\title{
High-precision analysis of multiple sulfur isotopes using NanoSIMS
}

\author{
Erik H. Hauri a,*, Dominic Papineau ${ }^{\mathrm{b}, 1}{ }^{\text {, Jianhua Wang }}{ }^{\mathrm{a}}$, Francois Hillion ${ }^{\mathrm{c}}$ \\ a Department of Terrestrial Magnetism, Carnegie Institution of Washington, Washington, DC 20015, United States \\ b Geophysical Laboratory, Carnegie Institution of Washington, Washington, DC 20015, United States \\ c Cameca SAS, 29, quai des Grésillons, 92230 Gennevilliers, France
}

\section{A R T I C L E I N F O}

\section{Article history:}

Received 13 April 2015

Received in revised form 11 November 2015

Accepted 14 November 2015

Available online 1 December 2015

\section{Keywords:}

Ion microprobe

Isotope geochemistry

Sulfur isotopes

Mass-independent fractionation

Mass spectrometery

NanoSIMS

Neoarchean

\begin{abstract}
A B S T R A C T
We report here data of multiple sulfur isotopes $\left({ }^{32} \mathrm{~S},{ }^{33} \mathrm{~S}\right.$, and $\left.{ }^{34} \mathrm{~S}\right)$ measured by NanoSIMS 50L ion microprobe in Fe-Ni-Cu standards, and pyrite grains recovered from the Neoarchean Abitibi Supracrustal Terrain, Canada. Careful attention to instrument tuning, sample preparation and sample stage positioning results in highly precise and accurate data for ${ }^{32} \mathrm{~S}-{ }^{33} \mathrm{~S}-{ }^{34} \mathrm{~S}$ similar to that of larger radius SIMS instruments. Our results using a multi-Faraday collection system demonstrate that the NanoSIMS 50L instrument is capable of high-precision measurements of multiple isotopes of sulfur capable of resolving mass-independent variations in ${ }^{33} \mathrm{~S}\left(\Delta^{33} \mathrm{~S}\right)$ on the order of $0.4 \%$ $(2 \sigma)$ with improvements possible with higher S count rates. Pyrite grains from the Abitibi Supracrustal terrane have a large range of $\delta^{34} \mathrm{~S}$ values between -9.6 and $+6.3 \%$, and corresponding $\Delta^{33} \mathrm{~S}$ values between -0.8 and $+1.5 \%$, consistent with other datasets from Neoarchean sedimentary rocks. The new data indicate the incorporation of atmospheric sulfur affected by mass-independent fractionation (MIF) of ${ }^{33} \mathrm{~S}$ into the Neoarchean marine sulfur cycle and point to subsequent sulfate reducing microorganisms at that time.
\end{abstract}

(c) 2015 Elsevier B.V. All rights reserved.

\section{Introduction}

Natural geochemical processes often result in the variability of the stable isotopes of elements, and these compositional variations have long been exploited to gain insight into the processes at work in the Earth's crust, mantle and atmosphere (Thiemens and Heidenreich, 1983; Valley et al., 1986; Farquhar et al., 2000; Valley and Cole, 2001; Ono et al., 2003) as well as processes operating in other planetary objects (Greenwood et al., 2000; Mittlefehldt et al., 2008; Thiemens, 1999) and indeed throughout the solar system and beyond (Clayton, 2003; Messenger et al., 2003; Mittlefehldt et al., 2008). Terrestrial variations in stable isotope compositions of certain elements can be small, requiring high-precision measurements using significant amounts of analyte (e.g. Ono et al., 2006). However when the available amount of sample is limited, or the physical domains of geochemical interest are small, microbeam methods are required to obtain the data of interest. This choice yields a trade-off between the high spatial resolution obtainable by microbeam analytical methods, and the precision achievable at that spatial resolution.

Multiple isotopes of sulfur have proven very useful in studies of the Earth's sulfur cycle and its variability through time, in particular in examining the interaction of the crust and atmosphere during the first

\footnotetext{
* Corresponding author.

E-mail address: ehauri@ciw.edu (E.H. Hauri).

1 Now at London Centre for Nanotechnology and Department of Earth Sciences, University College London, 17-19 Gower Street, London, WC1H 1AH, United Kingdom.
}

half of Earth's evolution. In particular, in situ analyses of the massindependent variability of ${ }^{32} \mathrm{~S},{ }^{33} \mathrm{~S}$ and ${ }^{34} \mathrm{~S}$ that results from photolytic reactions, compared to the mass-dependent fractionation of these isotopes that results from kinetic and equilibrium reactions, has been used as a powerful tracer for the recycling of atmospheric sulfur into the Earth's crust and deep mantle (Farquhar et al., 2002; Mojzsis et al., 2003; Papineau and Mojzsis, 2006; Papineau et al., 2005; Whitehouse et al., 2005; Kamber and Whitehouse, 2007; Cates and Mojzsis, 2006; Papineau et al., 2007; Phillippot et al., 2007; Xiao et al., 2010; Kozdon et al., 2010; Williford et al., 2011; Reuschel et al., 2012; Evans et al., 2014). SIMS analyses of Neoarchean sulfides have recently been combined with SQUID magnetic microscopy to distinguish sulfur fractionated by sulfate reducing microorganisms from diagenetic and metamorphic sulfur (Fischer et al., 2014). Analyses by SIMS (7f Geo, IMS 1270 , or 1280 ion probes) typically require a primary beam current between 0.3 and $5 \mathrm{nA}$, which yield a spot size of about 10 to $30 \mu \mathrm{m}$ and a crater depth of several microns.

The Cameca NanoSIMS 50L, a multicollector SIMS instrument designed for high spatial resolution, has also been used for sulfur isotope analyses applied to a range of geological problems, including tracing the activity of Paleoarchean sulfate reducing microorganisms in basalts from the Hoogenoeg Formation in the Barberton Greenstone Belt (McLoughlin et al., 2012) and in sandstones from the Strelley Pool Formation in the Pilbara Craton (Wacey et al., 2010, 2011). Sulfur isotope analyses by NanoSIMS have also recently been used to trace thermochemical sulfate reduction in Carboniferous bitumen (King et al., 2014). So far however, no reliable high-precision ${ }^{33} \mathrm{~S}$ data has 
been reported using the NanoSIMS. In this paper, we describe the results of efforts to measure the isotopic composition of multiple sulfur isotopes using the NanoSIMS 50L ion microprobe at the Carnegie Institution of Washington. We show for the first time that this instrument is capable of high-precision measurements of sulfur isotopes in terrestrial sulfide samples with precision and accuracy comparable to that of large radius SIMS instruments. We also demonstrate its utility by examining a small suite of sulfide minerals from metasedimentary rocks from the 2.7 Ga Deloro and Michipicoten Groups of the Abitibi Supracrustal Terrain in Ontario, Canada.

\section{Description and tuning of the NanoSIMS 50L}

The measurements reported in this paper were obtained with a NanoSIMS 50L ion microprobe at the Carnegie Institution of
Washington's Department of Terrestrial Magnetism (DTM) in 2008. The NanoSIMS 50L is a new-generation ion microprobe developed by Cameca Instruments SA (Fig. 1). Compared with the original NanoSIMS 50 instrument, the 50L consists of a larger-radius magnet (increased from $550 \mathrm{~mm}$ to $650 \mathrm{~mm}$ ), seven collectors, and the addition of Faraday Cups and associated amplification. The instrument consists of a primary optical column, where ion beams generated by cesium or oxygen sources are accelerated, collimated and focused at normal incidence onto a flat sample residing in a computer-controlled sample stage with $\pm 2 \mu \mathrm{m}$ position reproducibility. The final stages of primary ion beam focusing, and initial focusing of sputtered ions, are achieved by a common co-axial lens stack (EOW, EOP, EOS, Fig. 1). The location of this lens stack close to the sample surface $(\sim 400 \mu \mathrm{m}$ between sample surface and EOW lens) is an important design feature that permits the instrument to achieve very small probe diameters (minimum $30 \mathrm{~nm}$

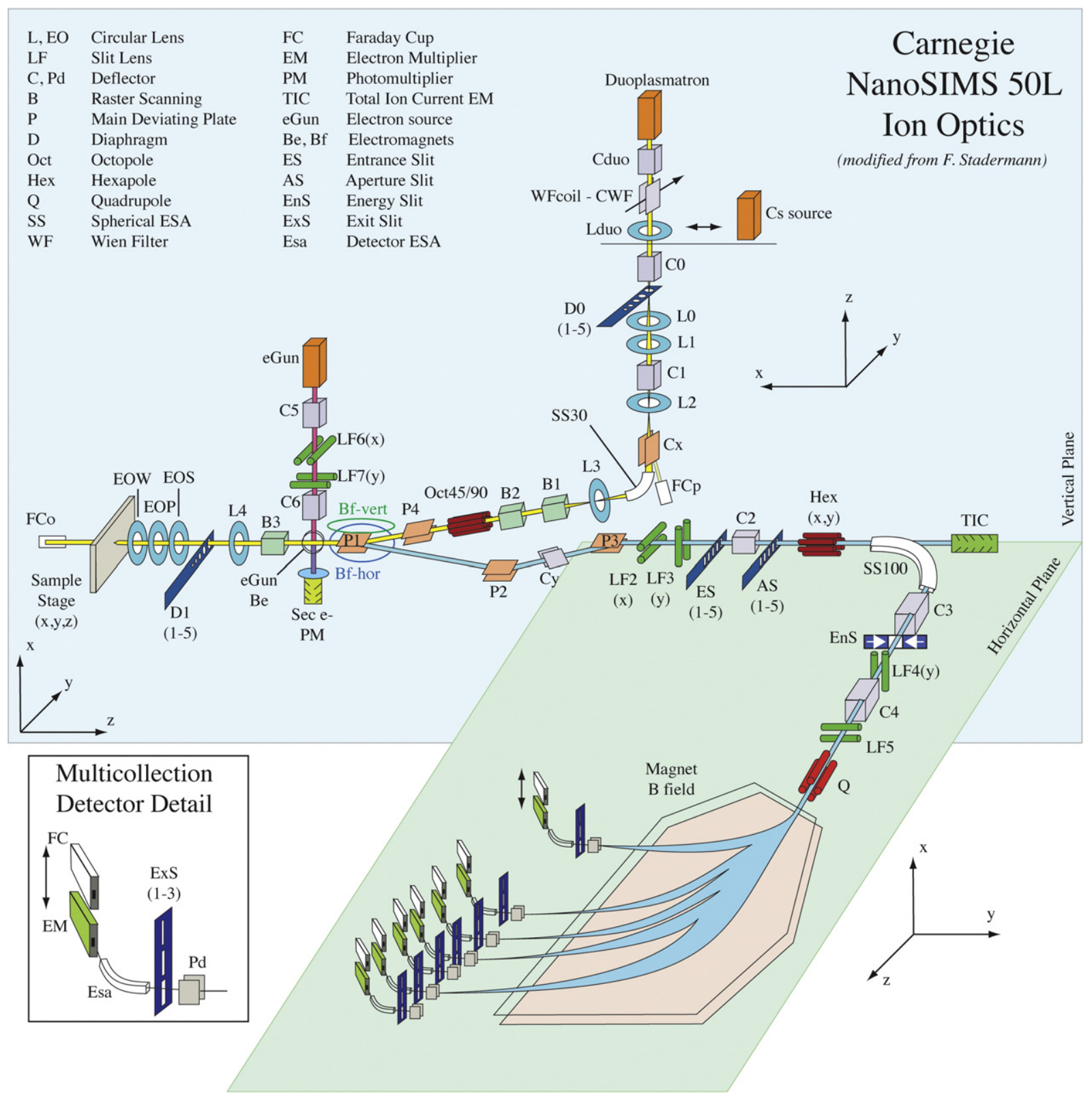

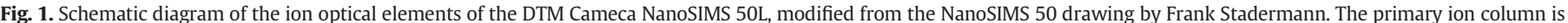

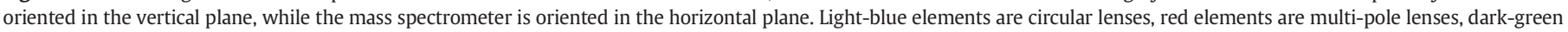

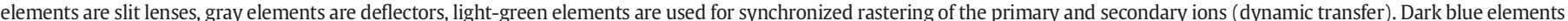

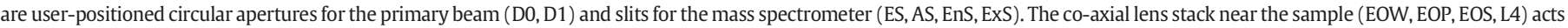
on both the primary and secondary ion beams, and requires the primary and secondary ions be of opposite polarity and equal energy. 
for $\mathrm{Cs}^{+}, 100 \mathrm{~nm}$ for $\mathrm{O}^{-}$). Sputtered secondary ions are focused into a mass spectrometer with a Mattauch-Herzog geometry, which permits the achievement of high mass resolving power and simultaneous collection of up to seven isotopic mass species across a wide mass range (factor of 22 from lowest mass to highest). The DTM instrument is the first NanoSIMS to be installed with both Faraday Cups and electron multipliers at each of its seven detectors and an evacuated temperature-controlled Faraday amplifier bin. The use of Faraday Cups connected to selectable resistors is a key feature of the instrument design that permits the acquisition of high-precision isotope data of the kind reported here.

For this project, we performed multicollection of ${ }^{32} \mathrm{~S},{ }^{33} \mathrm{~S}$ and ${ }^{34} \mathrm{~S}$ using Faraday Cups from various $\mathrm{Fe}-\mathrm{Ni}-\mathrm{Cu}$ sulfide minerals. $\mathrm{A} \mathrm{Cs}^{+}$ primary ion beam of 1-3 nA was focused to a diameter of $\sim 600 \mathrm{~nm}$; the focus of this primary beam tuning was optimized by observing a secondary electron image of an area of SiC grains $(<10 \mu \mathrm{m}$ in size) embedded in indium. The primary beam was rastered over an area $15 \mu \mathrm{m} \times 15 \mu \mathrm{m}$, divided into $64 \times 64$ pixels and a counting time of $245 \mu$ sec per pixel; although the response of the Faraday detector amplifier system is too slow to permit scanning ion imaging using Faraday Cups, this setup of the counting system effectively resulted in a scan time of $1 \mathrm{~s}$ per raster frame. The mass spectrometer was tuned to a mass resolving power of $\sim 7000$ (Cameca definition, using entrance slit \#4 and aperture slit \#3) sufficient to resolve isobaric interferences of sulfur hydrides and oxygen dimers from the sulfur peaks, and sufficient to obtain an intensity of $80-90 \mathrm{pA}\left(4.9\right.$ to $\left.5.5 \times 10^{8} \mathrm{cps}\right)$ of ${ }^{32} \mathrm{~S}^{-}$from pyrite. The NanoSIMS 50L contains a pair of Helmholz coils (Bf-hor and Bf-vert, Fig. 1) located outside the vacuum of the instrument in the transfer section in front of the entrance slit, in order to cancel out stray magnetic fields and prevent mass fractionation of the sputtered ions at the entrance slit to the mass spectrometer. During instrument tuning, these coils were carefully calibrated by examining horizontal and vertical scans of the ion beam in front of the entrance slit, using deflectors Cy, P2 and P3 (Fig. 1). For tuning of these coils, ${ }^{30} \mathrm{Si}^{-},{ }^{32} \mathrm{~S}^{-}$and ${ }^{35} \mathrm{Cl}^{-}$were sputtered from a silicon wafer and monitored on electron multipliers during these scans, and the Cy-P2/P3 centerline positions of each mass were determined for each scan. When the Helmholz coils are improperly tuned, each mass will show a slightly different centerline for Cy scan (corresponding to Bf-vert) or P2-P3 scan (corresponding to Bf-horiz). The Helmholz coil currents were tuned between scans, by checking both Bf-vert and Bf-horiz several times in succession, until all three isotopes gave the same Cy and P2-P3 centerlines within $1 \%$. For the initial tuning, we chose ${ }^{30} \mathrm{Si},{ }^{32} \mathrm{~S}$ and ${ }^{35} \mathrm{Cl}$ to tune the Helmholz coils because the wider mass range permits a precise tuning of these coils, and the chosen mass range is approximately centered on the sulfur isotope mass range of interest; a further detailed check was also done to ensure that measured sulfur isotope ratios were not overly sensitive to the position of the sputtered ion beam at the entrance slit. The Faraday measurement system used $1 \mathrm{E}+11 \Omega$ resistors and each Faraday amplifier was subject to gain calibration using a constant current source. After tuning of the primary optics, Helmholz coils and mass spectrometer for mass resolution, peak shape, peak flatness and Faraday amplifier gains, the instrument was then ready for measurement of multiple sulfur isotopes in standards and unknowns.

Prior to each sulfur isotope measurement, the analyzed area was pre-sputtered for three minutes, and then the sputtered ion beam was aligned horizontally and vertically in the entrance slit via automated scanning of $\mathrm{Cx}$ and $\mathrm{P} 2 / \mathrm{P} 3$ deflectors respectively. We collected 10 blocks of 40 ratios per block for a total of 400 ratio measurements, with offpeak measurement of Faraday Cup and electron multiplier baselines every 100 ratios, obtained by deflecting the sputtered ion beam out of the detectors using $-30 \mathrm{~V}$ deflection applied to the electrostatic deflectors in front of each detector's exit slit (Fig. 1), equivalent to $-0.1 \mathrm{amu}$ off the sulfur peaks. Off-peak baselines were typically $<0.01 \mathrm{pA}$. The total analysis time of $13 \mathrm{~min}$ resulted in a sputtered volume measuring $15 \mu \mathrm{m} \times 15 \mu \mathrm{m} \times 3 \mu \mathrm{m}$ or approximately $2.4 \mathrm{ng}$ of sulfur in common
FeCuNi sulfides. The useful ion yield (ions collected/atoms sputtered) was thus approximately $1-2 \%$ at a mass resolving power of 7000 (Cameca definition).

\section{Analytical methods}

\subsection{Standards and initial results}

Early measurements of sulfur isotopes in pyrite on this instrument in the Cameca factory, and later after installation in 2006, demonstrated

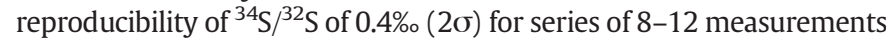
in a single $15 \times 15 \mu \mathrm{m}$ crater (Fig. $2 \mathrm{a}$ ). At the same time, these measurements displayed a time-dependent variation in the measured isotope ratio that was well correlated with crater depth, suggesting that improved control of measurement conditions could result in better performance; analysis of 10 different craters on a single grain of pyrite

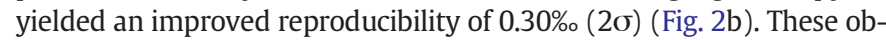
servations indicated clear avenues for further improvements in isotope ratio reproducibility, and justified our decision to equip our NanoSIMS 50L with Faraday cup measurement systems on all detectors. These
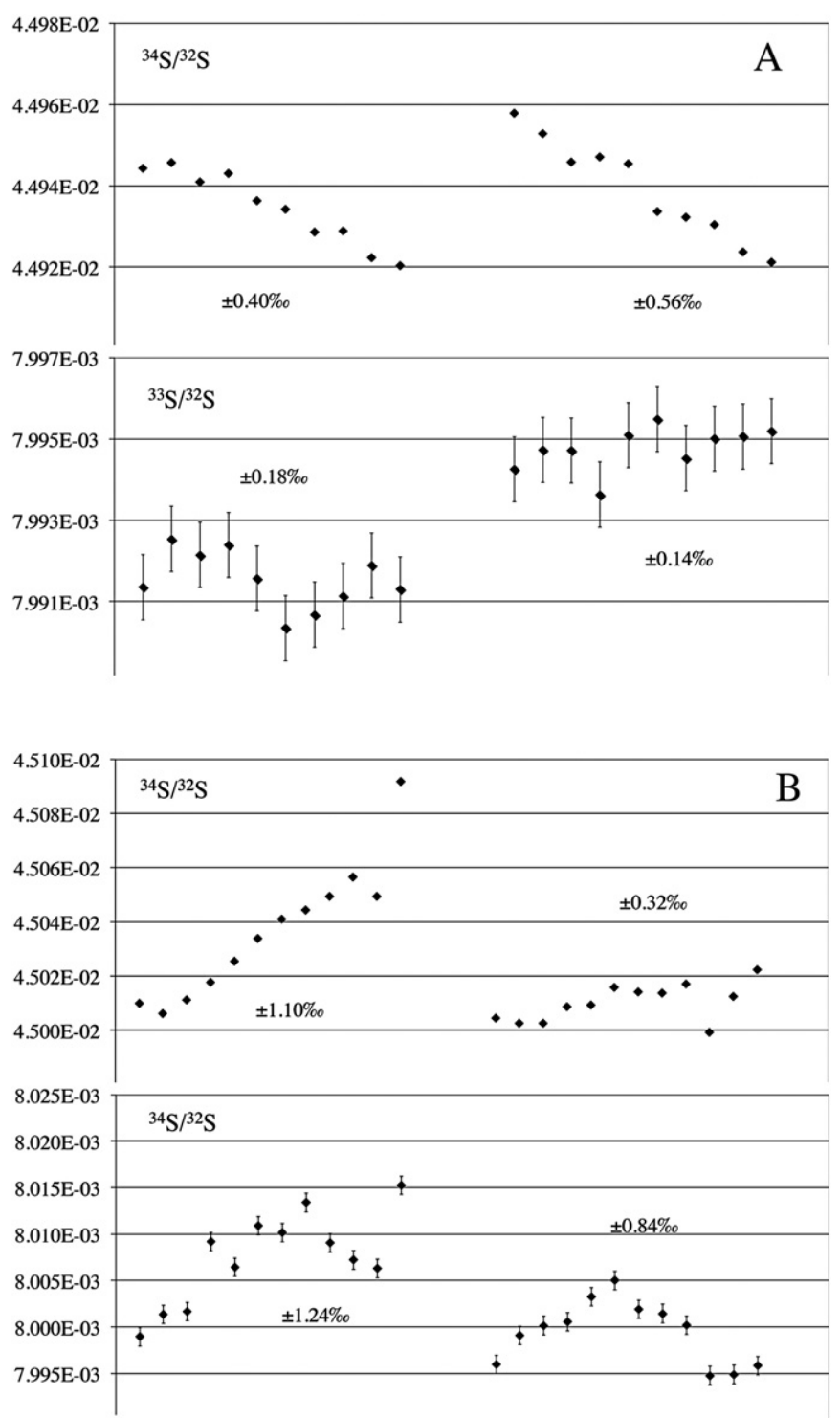

Fig. 2. Reproducibility of sulfur isotopes in a grain of Balmat pyrite, obtained from (A) repeat measurements within a single crater, and (B) from 10 separate craters. Errors are all $2 \sigma$. These early measurements, conducted in 2006, demonstrated the ability of the collection system of the NanoSIMS 50 L to obtain high-precision data. 
promising initial measurements led to the development effort that is the subject of this paper.

All of the data reported here were obtained in two analytical sessions, with only minor changes to the primary beam tuning between sessions (to optimize beam diameter). A later session, after minor changes to detector alignments, was used for calibration of matrix effects on SIMS instrumental mass fractionation. For SIMS standardization we utilized Canyon Diablo troilite (CDT), and the sulfides Balmat pyrite, Ruttan pyrite, Anderson pyrrhotite, Norilsk pentlandite and Trout Lake chalcopyrite described and analyzed by Crowe and Vaughan (1996) (note that Kozdon et al. (2010); Whitehouse (2013) and Cabral et al. (2013) report conventional data for Balmat pyrite that is 1\% higher than used here). Sample preparation simply involved grains pressed into indium metal filling $\sim 2 \mathrm{~mm}$ diameter holes drilled into $10 \mathrm{~mm}$ diameter aluminum stubs. The stubs were then polished down to $1 \mu \mathrm{m}$ diamond and coated with $20-30 \mathrm{~nm}$ of gold for SIMS work. CDT is known to be slightly heterogeneous at the $\sim 0.5 \%$ level (Ding et al., 2001), and the other standards are variably heterogeneous at the $0.2 \%$ o to $\sim 0.6 \%$ level, at the millimeter to $30 \mu \mathrm{m}$ scale, as demonstrated by several micro-drilling, laser-sampling and SIMS studies, with Balmat pyrite being the most homogeneous (Crowe and Vaughan, 1996; Paterson et al., 1997; Riciputi et al., 1998). Major element and sulfur isotopic compositions, relative to V-CDT, are given in Crowe and Vaughan (1996). In order to convert reported $\delta^{34} S$ values of our standards to absolute ${ }^{34} \mathrm{~S} /{ }^{32} \mathrm{~S}$ ratios, we used the ${ }^{34} \mathrm{~S} /{ }^{32} \mathrm{~S}$ value of 0.0441626 for $\mathrm{V}-\mathrm{CDT}$ determined by Ding et al. (2001). This resulted in the absolute ${ }^{34} \mathrm{~S} /{ }^{32} \mathrm{~S}$ values for our standards given in Table 1 .

\subsection{Data acquisition and internal precision}

Within each analysis, the data for ${ }^{32} \mathrm{~S},{ }^{33} \mathrm{~S}$ and ${ }^{34} \mathrm{~S}$ were corrected for relative detector gains determined from constant-current amplifier calibration (normally constant to within a few parts per million), and average off-peak baselines for each Faraday detector were then subtracted from the corresponding gain-corrected intensities. These were the only corrections to the measured data prior to calculation of isotope ratios. The intensities for ${ }^{33} \mathrm{~S}$ and ${ }^{34} \mathrm{~S}$ were normalized to ${ }^{32} \mathrm{~S}$ in order to calculate measured ratios of ${ }^{33} \mathrm{~S} /{ }^{32} \mathrm{~S}$ and ${ }^{34} \mathrm{~S} /{ }^{32} \mathrm{~S}$ for each measurement cycle. For integration times of $1 \mathrm{~s}$ per ratio and 400 total cycles, we obtained in-run precision $\left(2 \sigma_{\mathrm{M}}\right)$ of $0.16 \%$. for ${ }^{33} \mathrm{~S} /{ }^{32} \mathrm{~S}$, and $0.06 \%$ 。 for ${ }^{34} \mathrm{~S} /{ }^{32} \mathrm{~S}$ in an analysis time of $13 \mathrm{~min}$ (Table 2 ). These in-run precisions were similar to those expected from counting statistics and baseline subtraction uncertainties. Under these conditions, no resolvable drift in the measured standards was observed in either of the two analytical sessions. Point-to-point reproducibility of the ratio measurements was a function of specific analysis and sample conditions, and will be described more fully below.

\subsection{Mass fractionation in SIMS}

Measured ratios for the sulfide standards were variably shifted from the absolute ratios due to instrumental mass fractionation (IMF). The

Table 1

Absolute sulfur isotope ratios for standard sulfides used in this study.

\begin{tabular}{lcc}
\hline & ${ }^{33} \mathrm{~S} /{ }^{32} \mathrm{~S}^{1}$ & ${ }^{34} \mathrm{~S} /{ }^{32} \mathrm{~S}^{2}$ \\
\hline Canyon Diablo troilite & 0.0078842 & 0.0441626 \\
Balmat pyrite & 0.0079457 & 0.0448295 \\
Ruttan pyrite & 0.0078891 & 0.0442156 \\
Anderson pyrrhotite & 0.0078899 & 0.0442244 \\
Norilsk pentlandite & 0.0079164 & 0.0445115 \\
Trout Lake chalcopyrite & 0.0078854 & 0.0441758 \\
\hline
\end{tabular}

\footnotetext{
1 Value for CDT determined in this study, with all other values calculated from $\delta^{34} \mathrm{~S}$ dat of Crowe and Vaughan (1996) assuming a MDFL slope of 0.5167.

${ }^{2}$ Value for CDT from Ding et al. (2001) with all other values calculated from $\delta^{34} \mathrm{~S}$ data of Crowe and Vaughan (1996).
}

measured ${ }^{34} \mathrm{~S} /{ }^{32} \mathrm{~S}$ and ${ }^{33} \mathrm{~S} /{ }^{32} \mathrm{~S}$ ratios were corrected for IMF using an exponential law (Russell et al., 1978):

$$
\left({ }^{33} \mathrm{~S} /{ }^{32} \mathrm{~S}\right)_{\text {meas }} /\left({ }^{33} \mathrm{~S} /{ }^{32} \mathrm{~S}\right)_{\text {true }}=\left[\left({ }^{34} \mathrm{~S} /{ }^{32} \mathrm{~S}\right)_{\text {meas }} /\left({ }^{34} \mathrm{~S} /{ }^{32} \mathrm{~S}\right)_{\text {true }}\right]^{\beta_{\text {IMF }}}
$$

where the exponent $\beta_{I M F}$ must be chosen to accurately represent the fractionation behavior of the instrument being used. We have chosen to calculate $\beta_{I M F}$ using the expression commonly used for mass spectrometry (Hart and Zindler, 1989; Young et al., 2002):

$\beta_{\text {IMF }}=\ln (\mathrm{m} 33 / \mathrm{m} 32) / \ln (\mathrm{m} 34 / \mathrm{m} 32)=0.50831$

where $m_{i j}$ is the mass of the isotope of interest. This is not the only choice for calculating $\beta_{I M F}$; we will discuss this point further below.

In addition to the absolute ratios that we report, after correction for IMF the sulfur isotope compositions are also expressed in the conventional delta notation as the deviation, in parts per thousand (\%o), of the sample's sulfur isotope ratio from that of the V-CDT standard:

$\delta R(\%)=\left(R_{\text {sample }} / R_{\mathrm{V}-\mathrm{CDT}}-1\right) * 1000$

where $R_{\text {sample }}$ is the ratio $\left({ }^{34} \mathrm{~S} /{ }^{32} \mathrm{~S}\right.$ and $\left.{ }^{33} \mathrm{~S} /{ }^{32} \mathrm{~S}\right)$ determined for the sample and $R_{V-C D T}$ is the same ratio accepted for V-CDT (Ding et al., 2001). Natural mass-dependent variations in $\delta^{33} S$ and $\delta^{34} S$ are correlated along a mass-dependent fractionation line (MDFL), however one goal of this study is to evaluate the NanoSIMS for its ability to resolve mass-independent variations in ${ }^{33} \mathrm{~S}$ as expressed by the deviation of the abundances of these isotopes from the MDFL:

$\Delta^{33} \mathrm{~S}=\delta^{33} \mathrm{~S}_{\text {sample }}-1000 *\left[\left(1+\delta^{34} \mathrm{~S}_{\text {sample }} / 1000\right)^{\beta_{\mathrm{MDF}}}-1\right]$

where the exponent $\beta_{M D F L}$ is the slope of the sulfur isotope MDFL. For $\Delta^{33} \mathrm{~S}$ the value of $\beta_{M D F L}=0.515$ is most commonly employed (Farquhar et al., 2000; Farquhar and Wing, 2003). In addition to massindependent isotope shifts in ${ }^{33} \mathrm{~S}$, different equilibrium and kinetic processes that result in natural mass-dependent sulfur isotope fractionation can be characterized by slightly different values of $\beta_{\text {MDFL }}$ (Young et al., 2002).

Because the ion microprobe measures absolute sulfur isotope ratios rather than directly measuring deviations from a standard (as with conventional dual-inlet gas source mass spectrometers), the calculated magnitude of the IMF depends upon the chosen absolute values for VCDT. We have chosen the ${ }^{34} \mathrm{~S} /{ }^{32} \mathrm{~S}$ value determined by Ding et al. (2001) as we believe it to be currently the most accurate measurement of the absolute sulfur isotope composition of V-CDT for these two isotopes, as reflected from the high level of reproducibility of their measurements. On this scale, the IMF varied from nearly zero for Canyon Diablo troilite to $+3.7 \%$ /amu for Balmat pyrite, and was found to correlate with the $\mathrm{Fe} / \mathrm{S}$ ratio of the standards with some deviations dependent on $\mathrm{Ni}$ and $\mathrm{Cu}$ contents; the relative sense of these matrix effects are similar to those observed previously (Paterson et al., 1997; Riciputi et al., 1998), but the IMF magnitudes are different because we have chosen to use the most recent ratios for V-CDT (Ding et al., 2001) rather than the older values given by Thode et al. (1961) which are lower by 9\% per amu. It is important to point out that the choice of absolute ${ }^{34} \mathrm{~S} /{ }^{32} \mathrm{~S}$ ratio for $\mathrm{V}$-CDT has essentially no effect on calculated delta values, and as a result our delta values can be compared directly with prior studies without bias. We will, however, comment below on the most appropriate value for the ${ }^{33} \mathrm{~S} /{ }^{32} \mathrm{~S}$ ratio of $\mathrm{V}-\mathrm{CDT}$.

\subsection{Correction for IMF and calculation of $\Delta^{33} S$}

We examined two different methods for determining the exponent $\beta_{M D F L}$ and calculating $\Delta^{33} \mathrm{~S}$ values from our data. The Regression Method results in a single IMF correction factor for each mineral type, which is 
Table 2

NanoSIMS analyses of multiple sulfur isotopes $\left({ }^{32} \mathrm{~S},{ }^{33} \mathrm{~S}\right.$, and $\left.{ }^{34} \mathrm{~S}\right)$ in sulfide standards and in Neoarchaen Abitibi sulfides for analytical sessions 1 and 2.

\begin{tabular}{|c|c|c|c|c|c|}
\hline Analysis name & ${ }^{34} \mathrm{~S} /{ }^{32} \mathrm{~S}$ & ${ }^{33} \mathrm{~S} /{ }^{32} \mathrm{~S}$ & $\delta^{34} S_{\mathrm{CDT}}$ & $\delta^{33} \mathrm{~S}_{\mathrm{CDT}}$ & $\Delta^{33} S$ \\
\hline \multicolumn{6}{|l|}{ SESSION 1} \\
\hline \multicolumn{6}{|l|}{ CDT (Troilite) } \\
\hline $5 c-04 \_1$ & 0.044146 & 7.8815E-03 & -0.38 & -0.34 & -0.14 \\
\hline $5 c-04 \_2$ & 0.044151 & 7.8808E-03 & -0.27 & -0.42 & -0.29 \\
\hline 5c-04_3 & 0.044163 & 7.8834E-03 & +0.01 & -0.09 & -0.10 \\
\hline $5 c-04 \_3$ & 0.044163 & 7.8834E-03 & +0.01 & -0.09 & -0.10 \\
\hline 10c-01_1 & 0.044157 & 7.8813E-03 & -0.13 & -0.36 & -0.29 \\
\hline 10c-01_2 & 0.044175 & 7.8871E-03 & +0.28 & +0.38 & +0.23 \\
\hline 10c-01_3 & 0.044168 & 7.8855E-03 & +0.12 & +0.17 & +0.11 \\
\hline 10c-01_4 & 0.044167 & 7.8821E-03 & +0.10 & -0.26 & -0.31 \\
\hline 10c-01_5 & 0.044182 & 7.8855E-03 & +0.44 & +0.17 & -0.05 \\
\hline 10c-01_6 & 0.044191 & 7.8857E-03 & +0.64 & +0.20 & -0.13 \\
\hline 10c-01_7 & 0.044195 & 7.8871E-03 & +0.73 & +0.38 & 0.00 \\
\hline 10c-01_8 & 0.044106 & 7.8783E-03 & -1.28 & -0.74 & -0.08 \\
\hline 10c-01_9 & 0.044153 & 7.8815E-03 & -0.22 & -0.34 & -0.22 \\
\hline 10c-01_10 & 0.044161 & 7.8851E-03 & -0.04 & +0.12 & +0.14 \\
\hline \multicolumn{6}{|l|}{ Balmat (Pyrite) } \\
\hline NE-10c_1 & 0.044858 & 7.9465E-03 & +15.75 & +7.91 & -0.20 \\
\hline NE-10c_2 & 0.044831 & 7.9460E-03 & +15.14 & +7.85 & +0.05 \\
\hline NE-10c_3 & 0.044819 & 7.9425E-03 & +14.87 & +7.40 & -0.25 \\
\hline NE-10c_4 & 0.044833 & 7.9429E-03 & +15.18 & +7.45 & -0.36 \\
\hline NE-10c_5 & 0.044828 & 7.9453E-03 & +15.07 & +7.76 & 0.00 \\
\hline NE-10c_6 & 0.044843 & 7.9472E-03 & +15.41 & +8.00 & +0.06 \\
\hline NE-10c_7 & 0.044848 & 7.9461E-03 & +15.52 & +7.86 & -0.13 \\
\hline NE-10c_8 & 0.044850 & 7.9478E-03 & +15.57 & +8.07 & +0.06 \\
\hline NE-10c_9 & 0.044851 & 7.9471E-03 & +15.59 & +7.98 & -0.04 \\
\hline NE-10c_10 & 0.044864 & 7.9462E-03 & +15.88 & +7.87 & -0.30 \\
\hline Tour8c-02_1 & 0.044853 & 7.9496E-03 & +15.63 & +8.30 & +0.25 \\
\hline Tour8c-02_2 & 0.044859 & 7.9494E-03 & +15.77 & +8.27 & +0.16 \\
\hline Tour8c-02_3 & 0.044831 & 7.9468E-03 & +15.14 & +7.95 & +0.15 \\
\hline Tour8c-02_4 & 0.044831 & 7.9466E-03 & +15.14 & +7.92 & +0.13 \\
\hline Tour8c-02_5 & 0.044849 & 7.9491E-03 & +15.54 & +8.24 & +0.23 \\
\hline Tour8c-02_7 & 0.044845 & 7.9470E-03 & +15.45 & +7.97 & +0.02 \\
\hline Tour8c-02_8 & 0.044854 & 7.9476E-03 & +15.66 & +8.05 & -0.01 \\
\hline Tour8c-03_1 & 0.044827 & 7.9486E-03 & +15.05 & +8.17 & +0.43 \\
\hline Tour8c-03_2 & 0.044851 & 7.9469E-03 & +15.59 & +7.96 & -0.07 \\
\hline Tour8c-03_3 & 0.044828 & 7.9460E-03 & +15.07 & +7.85 & +0.09 \\
\hline Tour8c-03_4 & 0.044819 & 7.9464E-03 & +14.87 & +7.90 & +0.24 \\
\hline Tour8c-03_5 & 0.044829 & 7.9440E-03 & +15.09 & +7.59 & -0.18 \\
\hline Tour8c-03_7 & 0.044830 & 7.9470E-03 & +15.12 & +7.97 & +0.19 \\
\hline Tour8c-03_8 & 0.044834 & 7.9476E-03 & +15.21 & +8.05 & +0.22 \\
\hline Tour8c-04_1 & 0.044810 & 7.9442E-03 & +14.67 & +7.62 & +0.07 \\
\hline Tour8c-04_2 & 0.044847 & 7.9469E-03 & +15.50 & +7.96 & -0.02 \\
\hline Tour8c-04_3 & 0.044832 & 7.9472E-03 & +15.16 & +8.00 & +0.19 \\
\hline Tour8c-04_4 & 0.044797 & 7.9423E-03 & +14.38 & +7.36 & -0.04 \\
\hline Tour8c-04_5 & 0.044820 & 7.9427E-03 & +14.89 & +7.43 & -0.24 \\
\hline Tour8c-04_7 & 0.044852 & 7.9508E-03 & +15.61 & +8.45 & +0.42 \\
\hline Tour8c-04_8 & 0.044856 & 7.9465E-03 & +15.70 & +7.91 & -0.17 \\
\hline Tour8c-05_1 & 0.044837 & 7.9453E-03 & +15.27 & +7.76 & -0.11 \\
\hline Tour8c-05_2 & 0.044837 & 7.9462E-03 & +15.27 & +7.87 & +0.01 \\
\hline Tour8c-05_3 & 0.044839 & 7.9466E-03 & +15.32 & +7.92 & +0.03 \\
\hline Tour8c-05_4 & 0.044830 & 7.9471E-03 & +15.12 & +7.98 & +0.20 \\
\hline Tour8c-05_5 & 0.044836 & 7.9472E-03 & +15.25 & +8.00 & +0.14 \\
\hline Tour8c-05_7 & 0.044860 & $7.9480 \mathrm{E}-03$ & +15.79 & +8.10 & -0.03 \\
\hline Tour8c-05_8 & 0.044854 & 7.9481E-03 & +15.66 & +8.11 & +0.05 \\
\hline Tour8c-06_1 & 0.044803 & 7.9447E-03 & +14.51 & +7.68 & +0.21 \\
\hline Tour8c-06_2 & 0.044852 & 7.9463E-03 & +15.61 & +7.88 & -0.15 \\
\hline Tour8c-06_3 & 0.044849 & 7.9501E-03 & +15.54 & +8.36 & +0.36 \\
\hline Tour8c-06_4 & 0.044833 & 7.9454E-03 & +15.18 & +7.77 & -0.05 \\
\hline Tour8c-06_5 & 0.044859 & 7.9484E-03 & +15.77 & +8.15 & +0.03 \\
\hline Tour8c-06_7 & 0.044854 & 7.9480E-03 & +15.66 & +8.10 & +0.04 \\
\hline Tour8c-06_8 & 0.044847 & $7.9468 \mathrm{E}-03$ & +15.50 & +7.95 & -0.03 \\
\hline NE-10c-02_1 & 0.044858 & 7.9465E-03 & +15.75 & +7.91 & -0.20 \\
\hline NE-10c-02_2 & 0.044831 & 7.9460E-03 & +15.14 & +7.85 & +0.05 \\
\hline NE-10c-02_3 & 0.044819 & 7.9425E-03 & +14.87 & +7.40 & -0.25 \\
\hline NE-10c-02_4 & 0.044833 & 7.9429E-03 & +15.18 & +7.45 & -0.36 \\
\hline NE-10c-02_5 & 0.044828 & 7.9453E-03 & +15.07 & +7.76 & 0.00 \\
\hline NE-10c-02_6 & 0.044843 & 7.9472E-03 & +15.41 & +8.00 & +0.06 \\
\hline NE-10c-02_7 & 0.044848 & 7.9461E-03 & +15.52 & +7.86 & -0.13 \\
\hline NE-10c-02_8 & 0.044850 & $7.9478 \mathrm{E}-03$ & +15.57 & +8.07 & +0.06 \\
\hline NE-10c-02_9 & 0.044851 & $7.9471 \mathrm{E}-03$ & +15.59 & +7.98 & -0.04 \\
\hline NE-10c-02_10 & 0.044864 & 7.9462E-03 & +15.88 & +7.87 & -0.30 \\
\hline Tour12C-67_1 & 0.044860 & 7.9512E-03 & +15.79 & +8.50 & +0.37 \\
\hline Tour12C-67_2 & 0.044851 & 7.9464E-03 & +15.59 & +7.90 & -0.13 \\
\hline Balmat (Pyrite) & & & & & \\
\hline
\end{tabular}


Table 2 (continued)

\begin{tabular}{|c|c|c|c|c|c|}
\hline Analysis name & ${ }^{34} \mathrm{~S} /{ }^{32} \mathrm{~S}$ & ${ }^{33} \mathrm{~S} /{ }^{32} \mathrm{~S}$ & $\delta^{34} \mathrm{~S}_{\mathrm{CDT}}$ & $\delta^{33} \mathrm{~S}_{\mathrm{CDT}}$ & $\Delta^{33} S$ \\
\hline Tour12C-67_3 & 0.044847 & 7.9464E-03 & +15.50 & +7.90 & -0.08 \\
\hline Tour12C-67_4 & 0.044818 & 7.9484E-03 & +14.85 & +8.15 & +0.50 \\
\hline Tour12C-67_5 & 0.044843 & 7.9475E-03 & +15.41 & +8.03 & +0.10 \\
\hline Tour12C-67_6 & 0.044852 & 7.9470E-03 & +15.61 & +7.97 & -0.07 \\
\hline Tour12C-67_7 & 0.044852 & 7.9481E-03 & +15.61 & +8.11 & +0.07 \\
\hline Tour12C-67_8 & 0.044857 & 7.9471E-03 & +15.72 & +7.98 & -0.11 \\
\hline Tour12C-67_9 & 0.044832 & 7.9475E-03 & +15.16 & +8.03 & +0.23 \\
\hline Tour12C-67_10 & 0.044847 & 7.9471E-03 & +15.50 & +7.98 & +0.01 \\
\hline Tour12C-67_11 & 0.044855 & 7.9481E-03 & +15.68 & +8.11 & +0.04 \\
\hline Tour12C-67_12 & 0.044873 & 7.9480E-03 & +16.08 & +8.10 & -0.18 \\
\hline Tour12C-89_1 & 0.044778 & 7.9394E-03 & +13.93 & +7.00 & -0.17 \\
\hline Tour12C-89_2 & 0.044821 & 7.9460E-03 & +14.91 & +7.85 & +0.17 \\
\hline Tour12C-89_3 & 0.044829 & $7.9468 \mathrm{E}-03$ & +15.09 & +7.95 & +0.17 \\
\hline Tour12C-89_4 & 0.044830 & 7.9468E-03 & +15.12 & +7.95 & +0.16 \\
\hline Tour12C-89_5 & 0.044850 & 7.9463E-03 & +15.57 & +7.88 & -0.13 \\
\hline Tour12C-89_6 & 0.044849 & 7.9437E-03 & +15.54 & +7.55 & -0.45 \\
\hline Tour12C-89_7 & 0.044837 & 7.9459E-03 & +15.27 & +7.83 & -0.03 \\
\hline Tour12C-89_8 & 0.044843 & 7.9431E-03 & +15.41 & +7.48 & -0.45 \\
\hline Tour12C-89_9 & 0.044830 & 7.9459E-03 & +15.12 & +7.83 & +0.05 \\
\hline Tour12C-89_10 & 0.044835 & $7.9471 \mathrm{E}-03$ & +15.23 & +7.98 & +0.14 \\
\hline Tour12C-89_11 & 0.044860 & 7.9473E-03 & +15.79 & +8.01 & -0.12 \\
\hline Tour12C-89_12 & 0.044842 & 7.9453E-03 & +15.39 & +7.76 & -0.16 \\
\hline Tour12C-1011_1 & 0.044865 & 7.9465E-03 & +15.90 & +7.91 & -0.28 \\
\hline Tour12C-1011_2 & 0.044837 & $7.9451 \mathrm{E}-03$ & +15.27 & +7.73 & -0.13 \\
\hline Tour12C-1011_3 & 0.044843 & $7.9474 \mathrm{E}-03$ & +15.41 & +8.02 & +0.09 \\
\hline Tour12C-1011_4 & 0.044837 & 7.9461E-03 & +15.27 & +7.86 & -0.01 \\
\hline Tour12C-1011_5 & 0.044830 & 7.9452E-03 & +15.12 & +7.74 & -0.04 \\
\hline Tour12C-1011_6 & 0.044835 & 7.9457E-03 & +15.23 & +7.81 & -0.03 \\
\hline Tour12C-1011_7 & 0.044831 & 7.9457E-03 & +15.14 & +7.81 & +0.01 \\
\hline Tour12C-1011_8 & 0.044839 & 7.9489E-03 & +15.32 & +8.21 & +0.33 \\
\hline Tour12C-1011_9 & 0.044830 & 7.9467E-03 & +15.12 & +7.93 & +0.15 \\
\hline Tour12C-1011_10 & 0.044837 & $7.9416 \mathrm{E}-03$ & +15.27 & +7.28 & -0.59 \\
\hline Tour12C-1011_11 & 0.044848 & 7.9499E-03 & +15.52 & +8.34 & +0.35 \\
\hline Tour12C-1011_12 & 0.044853 & 7.9488E-03 & +15.63 & +8.20 & +0.15 \\
\hline Tour12C-1213_1 & 0.044803 & 7.9432E-03 & +14.51 & +7.49 & +0.02 \\
\hline Tour12C-1213_2 & 0.044823 & 7.9423E-03 & +14.96 & +7.36 & -0.34 \\
\hline Tour12C-1213_3 & 0.044843 & 7.9455E-03 & +15.41 & +7.78 & -0.15 \\
\hline Tour12C-1213_4 & 0.044818 & $7.9421 \mathrm{E}-03$ & +14.85 & +7.34 & -0.30 \\
\hline Tour12C-1213_5 & 0.044818 & $7.9453 \mathrm{E}-03$ & +14.85 & +7.76 & +0.11 \\
\hline Tour12C-1213_6 & 0.044821 & 7.9456E-03 & +14.91 & +7.79 & +0.12 \\
\hline Tour12C-1213_7 & 0.044816 & 7.9464E-03 & +14.80 & +7.90 & +0.27 \\
\hline Tour12C-1213_8 & 0.044824 & 7.9457E-03 & +14.98 & +7.81 & +0.09 \\
\hline Tour12C-1213_9 & 0.044838 & 7.9469E-03 & +15.30 & +7.96 & +0.08 \\
\hline Tour12C-1213_10 & 0.044836 & $7.9481 \mathrm{E}-03$ & +15.25 & +8.11 & +0.26 \\
\hline Tour12C-1213_11 & 0.044841 & $7.9454 \mathrm{E}-03$ & +15.36 & +7.77 & -0.14 \\
\hline Tour12C-1213_12 & 0.044847 & 7.9467E-03 & +15.50 & +7.93 & -0.05 \\
\hline Tour12C-1415_1 & 0.044801 & 7.9448E-03 & +14.47 & +7.69 & +0.25 \\
\hline Tour12C-1415_2 & 0.044833 & $7.9455 \mathrm{E}-03$ & +15.18 & +7.78 & -0.04 \\
\hline Tour12C-1415_3 & 0.044832 & 7.9448E-03 & +15.16 & +7.69 & -0.11 \\
\hline Tour12C-1415_4 & 0.044818 & 7.9427E-03 & +14.85 & +7.43 & -0.22 \\
\hline Tour12C-1415_5 & 0.044817 & 7.9423E-03 & +14.82 & +7.36 & -0.27 \\
\hline Tour12C-1415_6 & 0.044840 & 7.9472E-03 & +15.34 & +8.00 & +0.10 \\
\hline Tour12C-1415_7 & 0.044834 & 7.9447E-03 & +15.21 & +7.68 & -0.15 \\
\hline Tour12C-1415_8 & 0.044832 & 7.9433E-03 & +15.16 & +7.50 & -0.30 \\
\hline Tour12C-1415_9 & 0.044832 & $7.9460 \mathrm{E}-03$ & +15.16 & +7.85 & +0.04 \\
\hline Tour12C-1415_10 & 0.044846 & $7.9471 \mathrm{E}-03$ & +15.48 & +7.98 & +0.02 \\
\hline Tour12C-1415_11 & 0.044853 & 7.9468E-03 & +15.63 & +7.95 & -0.10 \\
\hline Tour12C-1415_12 & 0.044868 & 7.9509E-03 & +15.97 & +8.46 & +0.24 \\
\hline TourN-E01_1 & 0.044833 & 7.9449E-03 & +15.18 & +7.71 & -0.11 \\
\hline TourN-E01_2 & 0.044844 & 7.9462E-03 & +15.43 & +7.87 & -0.07 \\
\hline TourN-E01_3 & 0.044845 & 7.9465E-03 & +15.45 & +7.91 & -0.05 \\
\hline TourN-E01_4 & 0.044857 & 7.9470E-03 & +15.72 & +7.97 & -0.12 \\
\hline TourN-E01_5 & 0.044852 & $7.9478 \mathrm{E}-03$ & +15.61 & +8.07 & +0.04 \\
\hline TourN-E01_6 & 0.044857 & 7.9482E-03 & +15.72 & +8.12 & +0.03 \\
\hline TourN-E01_7 & 0.044852 & 7.9477E-03 & +15.61 & +8.06 & +0.02 \\
\hline TourN-E01_8 & 0.044855 & 7.9439E-03 & +15.68 & +7.58 & -0.49 \\
\hline TourN-E01_9 & 0.044859 & 7.9501E-03 & +15.77 & +8.36 & +0.25 \\
\hline TourN-E01_10 & 0.044842 & $7.9466 \mathrm{E}-03$ & +15.39 & +7.92 & 0.00 \\
\hline \multicolumn{6}{|l|}{ Ruttan (pyrite) } \\
\hline NW_1 & 0.044157 & 7.8815E-03 & -0.13 & -0.34 & -0.28 \\
\hline NW_2 & 0.044146 & $7.8821 \mathrm{E}-03$ & -0.37 & -0.27 & -0.08 \\
\hline NW_3 & 0.044147 & $7.8829 \mathrm{E}-03$ & -0.35 & -0.17 & +0.01 \\
\hline NW_4 & 0.044136 & 7.8819E-03 & -0.60 & -0.29 & +0.02 \\
\hline NW_5 & 0.044129 & 7.8793E-03 & -0.76 & -0.62 & -0.23 \\
\hline Tour10c-67_1 & 0.044173 & 7.8847E-03 & +0.23 & +0.06 & -0.06 \\
\hline
\end{tabular}


Table 2 (continued)

\begin{tabular}{|c|c|c|c|c|c|}
\hline Analysis name & ${ }^{34} \mathrm{~S} /{ }^{32} \mathrm{~S}$ & ${ }^{33} \mathrm{~S} /{ }^{32} \mathrm{~S}$ & $\delta^{34} \mathrm{~S}_{\mathrm{CDT}}$ & $\delta^{33} \mathrm{~S}_{\mathrm{CDT}}$ & $\Delta^{33} \mathrm{~S}$ \\
\hline Tour10c-67_2 & 0.044166 & 7.8835E-03 & +0.08 & -0.09 & -0.13 \\
\hline Tour10c-67_4 & 0.044141 & 7.8819E-03 & -0.49 & -0.29 & -0.04 \\
\hline Tour10c-67_7 & 0.044169 & 7.8864E-03 & +0.14 & +0.27 & +0.20 \\
\hline Tour10c-67_8 & 0.044188 & 7.8861E-03 & +0.57 & +0.24 & -0.06 \\
\hline Tour10c-67_9 & 0.044203 & 7.8876E-03 & +0.91 & +0.44 & -0.03 \\
\hline Tour10c-67_10 & 0.044178 & $7.8838 \mathrm{E}-03$ & +0.35 & -0.05 & -0.23 \\
\hline Tour10c-89_1 & 0.044166 & $7.8840 \mathrm{E}-03$ & +0.08 & -0.03 & -0.07 \\
\hline Tour10c-89_2 & 0.044156 & 7.8847E-03 & -0.15 & +0.06 & +0.14 \\
\hline Tour10c-89_4 & 0.044120 & 7.8794E-03 & -0.96 & -0.61 & -0.11 \\
\hline Tour10c-89_7 & 0.044164 & $7.8835 \mathrm{E}-03$ & +0.03 & -0.09 & -0.11 \\
\hline Tour10c-89_8 & 0.044196 & 7.8878E-03 & +0.75 & +0.46 & +0.08 \\
\hline Tour10c-89_9 & 0.044138 & $7.8818 \mathrm{E}-03$ & -0.55 & -0.31 & -0.02 \\
\hline Tour10c-89_10 & 0.044194 & 7.8876E-03 & +0.71 & +0.44 & +0.07 \\
\hline Tour10c-1011_1 & 0.044177 & 7.8877E-03 & +0.32 & +0.45 & +0.28 \\
\hline Tour10c-1011_2 & 0.044167 & 7.8852E-03 & +0.10 & +0.12 & +0.07 \\
\hline Tour10c-1011_4 & 0.044135 & 7.8877E-03 & -0.62 & +0.45 & +0.77 \\
\hline Tour10c-1011_7 & 0.044185 & $7.8865 \mathrm{E}-03$ & +0.50 & +0.29 & +0.03 \\
\hline Tour10c-1011_8 & 0.044178 & $7.8855 \mathrm{E}-03$ & +0.35 & +0.16 & -0.02 \\
\hline Tour10c-1011_9 & 0.044183 & $7.8848 \mathrm{E}-03$ & +0.46 & +0.07 & -0.16 \\
\hline Tour10c-1011_10 & 0.044188 & $7.8880 \mathrm{E}-03$ & +0.57 & +0.49 & +0.20 \\
\hline Tour10c-1213_1 & 0.044180 & 7.8861E-03 & +0.39 & +0.24 & +0.04 \\
\hline Tour10c-1213_2 & 0.044157 & $7.8841 \mathrm{E}-03$ & -0.13 & -0.02 & +0.05 \\
\hline Tour10c-1213_4 & 0.044133 & $7.8844 \mathrm{E}-03$ & -0.67 & +0.02 & +0.37 \\
\hline Tour10c-1213_7 & 0.044176 & $7.8856 \mathrm{E}-03$ & +0.30 & +0.17 & +0.02 \\
\hline Tour10c-1213_8 & 0.044194 & 7.8883E-03 & +0.71 & +0.53 & +0.16 \\
\hline Tour10c-1213_9 & 0.044205 & 7.8899E-03 & +0.95 & +0.73 & +0.24 \\
\hline Tour10c-1213_10 & 0.044199 & $7.8860 \mathrm{E}-03$ & +0.82 & +0.22 & -0.20 \\
\hline Tour10c-1415_1 & 0.044162 & $7.8854 \mathrm{E}-03$ & -0.01 & +0.15 & +0.16 \\
\hline Tour10c-1415_2 & 0.044157 & 7.8861E-03 & -0.13 & +0.24 & +0.30 \\
\hline Tour10c-1415_4 & 0.044152 & $7.8843 \mathrm{E}-03$ & -0.24 & +0.01 & +0.13 \\
\hline Tour10c-1415_7 & 0.044168 & $7.8836 \mathrm{E}-03$ & +0.12 & -0.08 & -0.14 \\
\hline Tour10c-1415_8 & 0.044177 & 7.8859E-03 & +0.32 & +0.21 & +0.04 \\
\hline Tour10c-1415_9 & 0.044188 & $7.8874 \mathrm{E}-03$ & +0.57 & +0.41 & +0.12 \\
\hline Tour10c-1415_10 & 0.044183 & 7.8847E-03 & +0.46 & +0.06 & -0.18 \\
\hline \multicolumn{6}{|l|}{ Anderson (pyrrhotite) } \\
\hline NPoN_2 & 0.044182 & 7.8849E-03 & +0.44 & +0.10 & -0.13 \\
\hline NPoN_3 & 0.044195 & $7.8876 \mathrm{E}-03$ & +0.73 & +0.44 & +0.06 \\
\hline NPoN_4 & 0.044207 & $7.8850 \mathrm{E}-03$ & +1.00 & +0.11 & -0.41 \\
\hline NPoN_5 & 0.044221 & 7.8872E-03 & +1.32 & +0.39 & -0.30 \\
\hline 10c-01_1 & 0.044214 & 7.8889E-03 & +1.16 & +0.60 & 0.00 \\
\hline 10c-01_2 & 0.044227 & 7.8928E-03 & +1.46 & +1.10 & +0.34 \\
\hline 10c-01_3 & 0.044225 & 7.8910E-03 & +1.41 & +0.87 & +0.14 \\
\hline 10c-01_4 & 0.044215 & 7.8881E-03 & +1.19 & +0.50 & -0.11 \\
\hline 10c-01_5 & 0.044233 & $7.8881 \mathrm{E}-03$ & +1.59 & +0.50 & -0.32 \\
\hline 10c-01_6 & 0.044255 & 7.8962E-03 & +2.09 & +1.53 & +0.45 \\
\hline 10c-01_7 & 0.044260 & $7.8926 \mathrm{E}-03$ & +2.20 & +1.07 & -0.07 \\
\hline 10c-01_8 & 0.044245 & 7.8920E-03 & +1.86 & +0.99 & +0.03 \\
\hline 10c-01_9 & 0.044243 & 7.8909E-03 & +1.82 & +0.86 & -0.08 \\
\hline 10c-01_10 & 0.044221 & $7.8858 \mathrm{E}-03$ & +1.32 & +0.21 & -0.47 \\
\hline \multicolumn{6}{|l|}{ Norilsk (pentlandite) } \\
\hline PtN_1 & 0.044542 & 7.9211E-03 & +8.59 & +4.69 & +0.26 \\
\hline PtN_2 & 0.044524 & 7.9135E-03 & +8.18 & +3.72 & -0.49 \\
\hline PtN_3 & 0.044526 & 7.9159E-03 & +8.22 & +4.03 & -0.21 \\
\hline PtN_4 & 0.044467 & 7.9129E-03 & +6.89 & +3.65 & +0.09 \\
\hline PtN_5 & 0.044517 & 7.9168E-03 & +8.02 & +4.14 & +0.01 \\
\hline 10c-01_1 & 0.044469 & 7.9152E-03 & +6.94 & +3.94 & +0.36 \\
\hline 10c-01_2 & 0.044531 & 7.9129E-03 & +8.34 & +3.65 & -0.65 \\
\hline 10c-01_3 & 0.044479 & 7.9143E-03 & +7.16 & +3.83 & +0.13 \\
\hline 10c-01_4 & 0.044514 & 7.9179E-03 & +7.95 & +4.28 & +0.18 \\
\hline 10c-01_5 & 0.044479 & $7.9156 \mathrm{E}-03$ & +7.16 & +3.99 & +0.30 \\
\hline 10c-01_6 & 0.044514 & 7.9183E-03 & +7.95 & +4.33 & +0.23 \\
\hline 10c-01_7 & 0.044510 & $7.9145 \mathrm{E}-03$ & +7.86 & +3.85 & -0.20 \\
\hline 10c-01_8 & 0.044570 & 7.9209E-03 & +9.22 & +4.66 & -0.09 \\
\hline 10c-01_9 & 0.044522 & 7.9174E-03 & +8.13 & +4.22 & +0.02 \\
\hline 10c-01_10 & 0.044511 & 7.9178E-03 & +7.88 & +4.27 & +0.20 \\
\hline \multicolumn{6}{|c|}{ Neoarchean sulfides (all pyrite) } \\
\hline MI0701 & 0.044443 & 7.9042E-03 & +6.35 & +2.54 & -0.74 \\
\hline MI0704 & 0.044354 & 7.9129E-03 & +4.33 & +3.64 & +1.40 \\
\hline MI0704 & 0.044332 & 7.9069E-03 & +3.83 & +2.88 & +0.90 \\
\hline MI0705 & 0.043797 & 7.8441E-03 & -8.29 & -5.08 & -0.79 \\
\hline TI0703 & 0.043744 & 7.8454E-03 & -9.48 & -4.92 & -0.01 \\
\hline \multicolumn{6}{|l|}{ SESSION 2} \\
\hline \multicolumn{6}{|l|}{ Balmat (pyrite) } \\
\hline NE-10c_1 & 0.044859 & 7.9467E-03 & +15.76 & +8.03 & -0.09 \\
\hline NE-10c_2 & 0.044846 & 7.9475E-03 & +15.47 & +8.13 & +0.16 \\
\hline Balmat (pyrite) & & & & & \\
\hline
\end{tabular}


Table 2 (continued)

\begin{tabular}{|c|c|c|c|c|c|}
\hline Analysis name & ${ }^{34} \mathrm{~S} /{ }^{32} \mathrm{~S}$ & ${ }^{33} \mathrm{~S} /{ }^{32} \mathrm{~S}$ & $\delta^{34} \mathrm{~S}_{\mathrm{CDT}}$ & $\delta^{33} \mathrm{~S}_{\mathrm{CDT}}$ & $\Delta^{33} S$ \\
\hline NE-10c_3 & 0.044827 & 7.9457E-03 & +15.04 & +7.90 & +0.15 \\
\hline NE-10c_4 & 0.044838 & 7.9452E-03 & +15.29 & +7.84 & -0.04 \\
\hline NE-10c_5 & 0.044852 & 7.9473E-03 & +15.60 & +8.10 & +0.06 \\
\hline NE-10c_6 & 0.044850 & 7.9440E-03 & +15.56 & +7.69 & -0.33 \\
\hline NE-10c_7 & 0.044842 & 7.9476E-03 & +15.38 & +8.14 & +0.22 \\
\hline NE-10c_8 & 0.044861 & 7.9473E-03 & +15.81 & +8.10 & -0.04 \\
\hline NE-10c_9 & 0.044864 & 7.9488E-03 & +15.87 & +8.29 & +0.11 \\
\hline NE-10c_10 & 0.044881 & 7.9502E-03 & +16.28 & +8.47 & +0.08 \\
\hline Tour8c-02_1 & 0.044880 & 7.9490E-03 & +16.25 & +8.32 & -0.06 \\
\hline Tour8c-02_2 & 0.044875 & 7.9476E-03 & +16.14 & +8.14 & -0.18 \\
\hline Tour8c-02_3 & 0.044864 & 7.9462E-03 & +15.87 & +7.96 & -0.21 \\
\hline Tour8c-02_4 & 0.044873 & 7.9489E-03 & +16.10 & +8.31 & +0.01 \\
\hline Tour8c-02_5 & 0.044860 & 7.9484E-03 & +15.78 & +8.24 & +0.11 \\
\hline Tour8c-02_7 & 0.044863 & 7.9459E-03 & +15.85 & +7.93 & -0.24 \\
\hline Tour8c-02_8 & 0.044814 & 7.9413E-03 & +14.75 & +7.35 & -0.26 \\
\hline Tour8c-03_1 & 0.044817 & 7.9445E-03 & +14.82 & +7.75 & +0.11 \\
\hline Tour8c-03_2 & 0.044836 & 7.9462E-03 & +15.24 & +7.96 & +0.11 \\
\hline Tour8c-03_3 & 0.044843 & 7.9467E-03 & +15.40 & +8.03 & +0.09 \\
\hline Tour8c-03_4 & 0.044841 & 7.9452E-03 & +15.36 & +7.84 & -0.08 \\
\hline Tour8c-03_5 & 0.044835 & $7.9445 \mathrm{E}-03$ & +15.22 & +7.75 & -0.09 \\
\hline Tour8c-03_7 & 0.044831 & 7.9439E-03 & +15.13 & +7.67 & -0.12 \\
\hline Tour8c-03_8 & 0.044831 & $7.9465 \mathrm{E}-03$ & +15.13 & +8.00 & +0.20 \\
\hline Tour8c-04_1 & 0.044827 & 7.9438E-03 & +15.04 & +7.66 & -0.09 \\
\hline Tour8c-04_2 & 0.044829 & 7.9458E-03 & +15.09 & +7.91 & +0.14 \\
\hline \multicolumn{6}{|l|}{ Ruttan (Pyrite) } \\
\hline$\overline{N W \_1}$ & 0.044206 & 7.8864E-03 & +0.99 & +0.39 & -0.13 \\
\hline NW_2 & 0.044189 & $7.8846 \mathrm{E}-03$ & +0.59 & +0.16 & -0.14 \\
\hline NW_3 & 0.044182 & $7.8860 \mathrm{E}-03$ & +0.43 & +0.34 & +0.11 \\
\hline NW_4 & 0.044189 & $7.8856 \mathrm{E}-03$ & +0.59 & +0.29 & -0.02 \\
\hline NW_5 & 0.044185 & 7.8867E-03 & +0.50 & +0.43 & +0.17 \\
\hline Tour10c-67_1 & 0.044190 & 7.8886E-03 & +0.63 & +0.67 & +0.34 \\
\hline Tour10c-67_2 & 0.044199 & 7.8876E-03 & +0.83 & +0.54 & +0.11 \\
\hline Tour10c-67_4 & 0.044194 & $7.8845 \mathrm{E}-03$ & +0.72 & +0.13 & -0.24 \\
\hline Tour10c-67_7 & 0.044205 & 7.8862E-03 & +0.97 & +0.36 & -0.14 \\
\hline Tour10c-67_8 & 0.044202 & 7.8879E-03 & +0.90 & +0.58 & +0.11 \\
\hline Tour10c-67_9 & 0.044160 & $7.8855 \mathrm{E}-03$ & -0.06 & +0.27 & +0.31 \\
\hline Tour10c-67_10 & 0.044161 & $7.8850 \mathrm{E}-03$ & -0.04 & +0.21 & +0.23 \\
\hline Tour10c-89_1 & 0.044144 & $7.8806 \mathrm{E}-03$ & -0.42 & -0.36 & -0.14 \\
\hline Tour10c-89_2 & 0.044140 & 7.8809E-03 & -0.51 & -0.32 & -0.05 \\
\hline Tour10c-89_4 & 0.044138 & 7.8804E-03 & -0.56 & -0.38 & -0.09 \\
\hline Tour10c-89_7 & 0.044137 & $7.8818 \mathrm{E}-03$ & -0.58 & -0.21 & +0.09 \\
\hline Tour10c-89_8 & 0.044132 & $7.8808 \mathrm{E}-03$ & -0.69 & -0.33 & +0.03 \\
\hline Tour10c-89_9 & 0.044136 & 7.8803E-03 & -0.60 & -0.40 & -0.08 \\
\hline Tour10c-89_10 & 0.044162 & 7.8824E-03 & -0.02 & -0.13 & -0.12 \\
\hline Tour10c-1011_1 & 0.044161 & $7.8828 \mathrm{E}-03$ & -0.04 & -0.08 & -0.06 \\
\hline \multicolumn{6}{|c|}{ Anderson (pyrrhotite) } \\
\hline NPoN_2 & 0.044263 & 7.8945E-03 & +2.27 & +1.41 & +0.24 \\
\hline NPoN_3 & 0.044235 & 7.8879E-03 & +1.64 & +0.58 & -0.27 \\
\hline NPoN_4 & 0.044241 & 7.8909E-03 & +1.77 & +0.96 & +0.04 \\
\hline NPoN_5 & 0.044243 & $7.8908 \mathrm{E}-03$ & +1.82 & +0.94 & 0.00 \\
\hline 10c-01_1 & 0.044211 & $7.8886 \mathrm{E}-03$ & +1.10 & +0.66 & +0.10 \\
\hline 10c-01_2 & 0.044208 & 7.8863E-03 & +1.03 & +0.37 & -0.16 \\
\hline $10 c-01 \_3$ & 0.044213 & $7.8862 \mathrm{E}-03$ & +1.14 & +0.36 & -0.23 \\
\hline 10c-01_4 & 0.044215 & $7.8854 \mathrm{E}-03$ & +1.19 & +0.26 & -0.35 \\
\hline 10c-01_5 & 0.044215 & $7.8885 \mathrm{E}-03$ & +1.19 & +0.65 & +0.04 \\
\hline 10c-01_6 & 0.044201 & $7.8868 \mathrm{E}-03$ & +0.87 & +0.44 & -0.01 \\
\hline \multicolumn{6}{|c|}{ Norilsk (pentlandite) } \\
\hline PtN_1 & 0.044464 & 7.9105E-03 & +6.83 & +3.43 & -0.09 \\
\hline PtN_2 & 0.044484 & 7.9139E-03 & +7.28 & +3.86 & +0.10 \\
\hline PtN_3 & 0.044537 & 7.9229E-03 & +8.48 & +5.02 & +0.64 \\
\hline PtN_4 & 0.044568 & 7.9202E-03 & +9.18 & +4.67 & -0.06 \\
\hline PtN_5 & 0.044561 & 7.9199E-03 & +9.02 & +4.64 & -0.02 \\
\hline 10c-01_1 & 0.044487 & 7.9095E-03 & +7.35 & +3.31 & -0.49 \\
\hline 10c-01_2 & 0.044474 & 7.9104E-03 & +7.06 & +3.42 & -0.22 \\
\hline 10c-01_3 & 0.044547 & 7.9204E-03 & +8.70 & +4.70 & +0.21 \\
\hline 10c-01_4 & 0.044491 & $7.9156 \mathrm{E}-03$ & +7.44 & +4.08 & +0.24 \\
\hline 10c-01_5 & 0.044501 & 7.9191E-03 & +7.67 & +4.54 & +0.58 \\
\hline \multicolumn{6}{|c|}{ Unknowns (pyrite) } \\
\hline \multicolumn{6}{|c|}{ Balmat (Wedge topography) } \\
\hline SWedge_1 & 0.044895 & 7.9504E-03 & +16.59 & +8.49 & -0.05 \\
\hline SWedge_2 & 0.044904 & 7.9480E-03 & +16.79 & +8.19 & -0.46 \\
\hline SWedge_3 & 0.044891 & 7.9499E-03 & +16.50 & +8.43 & -0.07 \\
\hline SWedge_4 & 0.044922 & $7.9476 \mathrm{E}-03$ & +17.20 & +8.14 & -0.72 \\
\hline SWedge_5 & 0.044980 & $7.9550 \mathrm{E}-03$ & +18.50 & +9.09 & -0.44 \\
\hline SWedge_6 & 0.044988 & 7.9609E-03 & +18.70 & +9.83 & +0.20 \\
\hline
\end{tabular}


Table 2 (continued)

\begin{tabular}{|c|c|c|c|c|c|}
\hline Analysis name & ${ }^{34} \mathrm{~S} /{ }^{32} \mathrm{~S}$ & ${ }^{33} \mathrm{~S} /{ }^{32} \mathrm{~S}$ & $\delta^{34} \mathrm{~S}_{\mathrm{CDT}}$ & $\delta^{33} \mathrm{~S}_{\mathrm{CDT}}$ & $\Delta^{33} S$ \\
\hline SWedge_7 & 0.044934 & 7.9535E-03 & +17.47 & +8.90 & -0.10 \\
\hline SWedge_8 & 0.044894 & 7.9509E-03 & +16.57 & +8.56 & +0.02 \\
\hline SWedge_9 & 0.044883 & 7.9506E-03 & +16.32 & +8.52 & +0.11 \\
\hline SWedge_10 & 0.044889 & 7.9517E-03 & +16.46 & +8.66 & +0.18 \\
\hline \multicolumn{6}{|c|}{ Ruttan (poor polish quality) } \\
\hline SWpoor_1 & 0.044011 & 7.8685E-03 & -3.43 & -1.89 & -0.11 \\
\hline SWpoor_2 & 0.044003 & $7.8728 \mathrm{E}-03$ & -3.61 & -1.34 & +0.53 \\
\hline SWpoor_3 & 0.043993 & 7.8673E-03 & -3.83 & -2.04 & -0.05 \\
\hline SWpoor_4 & 0.043996 & $7.8684 \mathrm{E}-03$ & -3.77 & -1.90 & +0.05 \\
\hline SWpoor_5 & 0.043990 & $7.8668 \mathrm{E}-03$ & -3.90 & -2.10 & -0.08 \\
\hline SWpoor_6 & 0.043974 & 7.8667E-03 & -4.26 & -2.11 & +0.09 \\
\hline SWpoor_7 & 0.043959 & 7.8636E-03 & -4.62 & -2.50 & -0.11 \\
\hline SWpoor_8 & 0.043952 & 7.8663E-03 & -4.78 & -2.16 & +0.31 \\
\hline SWpoor_9 & 0.043963 & 7.8627E-03 & -4.53 & -2.62 & -0.27 \\
\hline SWpoor_10 & 0.043966 & 7.8627E-03 & -4.44 & -2.62 & -0.32 \\
\hline \multicolumn{6}{|c|}{ Neoarchean sulfides (pyrite) } \\
\hline MI0701 & 0.044419 & $7.9046 \mathrm{E}-03$ & +5.82 & +2.69 & -0.32 \\
\hline MI0704 & 0.044343 & 7.9117E-03 & +4.09 & +3.59 & +1.48 \\
\hline MI0704 & 0.044349 & $7.9096 \mathrm{E}-03$ & +4.22 & +3.33 & +1.15 \\
\hline MI0705 & 0.043744 & $7.8408 \mathrm{E}-03$ & -9.47 & -5.40 & -0.49 \\
\hline TI0703 & 0.043736 & $7.8406 \mathrm{E}-03$ & -9.65 & -5.42 & -0.42 \\
\hline
\end{tabular}

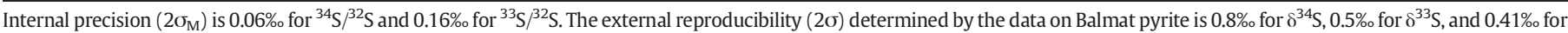
$\Delta^{33} \mathrm{~S}$. All delta values and associated uncertainties are in permil and normalized with respect to V-CDT.

then applied to unknowns of the same mineral type using Eqs. (1) and (2). The ${ }^{34} \mathrm{~S}$ Normalization Method uses the difference between the measured and "true" values of the ${ }^{34} \mathrm{~S} /{ }^{32} \mathrm{~S}$ ratio to calculate a separate IMF factor for each individual analysis, and unknowns are corrected using average IMF values for standard analyses that bracket them in time (again using Eqs. (1) and (2)). Comparison of the two methods demonstrates that they result in the same delta values and reproducibility to better than $0.01 \%$, and below we describe them both.

\subsubsection{Regression Method}

This method was described in detail by Papineau et al. (2005). For this method, we compiled all the measured ratios, grouped the data by mineral type and calculated an average IMF value for each mineral type (troilite, pyrite, pentlandite, chalcopyrite), defined as the ratio of the averaged measured ratio to the "true" ratio:

$\alpha_{\mathrm{IMF}}=(34 / 32)_{\text {meas }} /(34 / 32)_{\text {true }}$

and similarly the IMF value for ${ }^{33} \mathrm{~S} /{ }^{32} \mathrm{~S}$ was calculated as:

$\alpha(33)_{\mathrm{IMF}}=\left[\alpha(34)_{\mathrm{IMF}}\right]^{\beta_{\mathrm{IMF}}}$

where $\beta_{I M F}$ is as described above (Eq. (2)); see also Eqs. (19)-(21) in Young et al. (2002). The average $\alpha(34)_{\text {IMF }}$ and $\alpha(33)_{\text {IMF }}$ values thus calculated for each phase were then applied to the measured ratios for that phase in order to calculate fractionation-corrected ${ }^{34} \mathrm{~S} /{ }^{32} \mathrm{~S}$ and ${ }^{33} \mathrm{~S} /{ }^{32} \mathrm{~S}$ ratios (Eqs. (5), (6) and (2)). The IMF-corrected ratios for all phases were then regressed against each other on a $\mathrm{LN}-\mathrm{LN}$ basis, employing a regression for $\ln \left({ }^{34} \mathrm{~S} /{ }^{32} \mathrm{~S}\right)$ and $\ln \left({ }^{33} \mathrm{~S} /{ }^{32} \mathrm{~S}\right)$ using a full York regression including error propagation on both ratios (Mahon, 1996); the slope of this regression $(m)$ gives the slope of the mass dependent fractionation line $\left(\beta_{\text {MDFL }}\right)$ and the absolute ${ }^{33} \mathrm{~S} /{ }^{32} \mathrm{~S}$ ratio for $\mathrm{V}$-CDT is obtained from the Y-intercept (b33) using the expression:

${ }^{33} \mathrm{~S} /{ }^{32} \mathrm{~S}_{\mathrm{V}-\mathrm{CDT}}={ }^{34} \mathrm{~S} /{ }^{32} \mathrm{~S}_{\mathrm{V}-\mathrm{CDT}}{ }^{\left[{ }_{\mathrm{MDFL}} *(b 33)\right]}$

Under optimal conditions of instrument tuning and sample preparation, the regressed sulfur isotope MDFL slope for the $\delta^{33} \mathrm{~S}-\delta^{34} \mathrm{~S}$ correlation was $0.5167( \pm 0.00432 \sigma)$, within error of the theoretical equilibrium value of 0.5159 where $\beta_{\text {MDFL }}=(1 / \mathrm{m} 32-1 / \mathrm{m} 33) /(1 / \mathrm{m} 32-1 / \mathrm{m} 34)$ ((Young et al., 2002)). The average absolute value of ${ }^{33} \mathrm{~S} /{ }^{32} \mathrm{~S}$ for $\mathrm{V}-\mathrm{CDT}$ determined in this study was 0.00788419 ; the errors on the Y-intercept from the York regression result in an error estimate of $\pm 9 \%$ 。 $(2 \sigma)$ on this value, however this large error estimate is the result of an increase in the error envelope of the MDFL slope over a long extrapolation 3 orders of magnitude beyond the range of the data in LN-LN space. In reality the value of this ratio can be varied by only $\pm 0.46 \%$ 。 $(2 \sigma)$, propagating the error bounds on the MDFL slope, before the average value of $\Delta^{33} \mathrm{~S}$ for the standard data exceeds its $2 \sigma$ error bound. The value of 0.00788419 (363) is similar to the values obtained with a Cameca IMS 1270 ion microprobe by Papineau et al. (2005), but is $0.9 \%$ o higher than the value recommended by Ding et al. (2001) which itself has an uncertainty of $0.74 \%$ 。 $(2 \sigma)$; at this level, differences in collector gains between instruments, or so-called "Faraday cup factors" for any single instrument (which are not accounted for by Faraday amplifier gain calibration) could account for the difference in $\mathrm{V}-\mathrm{CDT}{ }^{33} \mathrm{~S} /{ }^{32} \mathrm{~S}$ values between this study and that of Ding et al. (2001). However, we wish to point out that if one uses our value for ${ }^{33} \mathrm{~S} /{ }^{32} \mathrm{~S}$, and the Ding et al. (2001) value for ${ }^{34} \mathrm{~S} /{ }^{32} \mathrm{~S}$, these ratios are related to the classic values of Thode et al. (1961) by simple exponential mass fractionation with a slope of 0.50828 , which is nearly identical to the slope used for mass spectrometry fractionation corrections (Eq. (2)).

This method, where the MDFL slope is determined by the quality of standard analyses within a given analytical session, has the effect of normalizing out any instrument-related vagaries that could in principal cause the data to deviate slightly from the theoretical MDFL slope, and eliminates the possibility of calculating "artificial" mass-independent $\Delta^{33} \mathrm{~S}$ anomalies under the assumption that the analytical instrument always perfectly reproduces any specific theoretical MDFL relationship. Using this method, average mean $\Delta^{33} \mathrm{~S}$ values measured for CDT which were not included in the regression were always within $2 \sigma$ of $0 \%$ for all analytical sessions. Average $2 \sigma$ reproducibility of $\Delta^{33} \mathrm{~S}$ values was $0.41 \%$ as determined from the standard deviation of individual $\Delta^{33} \mathrm{~S}$ standard values within the analytical session.

\subsubsection{The ${ }^{34} \mathrm{~S}$ normalization method}

For $\Delta^{33} \mathrm{~S}$ calculation using this method, the difference between the measured ${ }^{34} \mathrm{~S} /{ }^{32} \mathrm{~S}$ ratio for a standard and the "true" or known ratio gives a measure of the IMF for each individual standard analysis, and this measure of the IMF is used to calculate a fractionation-corrected ${ }^{33} \mathrm{~S} /{ }^{32} \mathrm{~S}$ ratio using Eqs. (1) and (2) and delta values using Eqs. (3) and (4). Calculations for unknowns are made using the same IMF corrections 
as standards that bracket them in time. Eqs. (3) and (4) are insensitive to the actual value of $\beta_{M D F L}$ used, so long as the same value is used to calculate absolute ratios for ${ }^{33} \mathrm{~S} /{ }^{32} \mathrm{~S}$ from $\mathrm{V}-\mathrm{CDT}$ and the delta values for the standards. This method assumes - probably incorrectly - that all measured variations of ${ }^{34} \mathrm{~S} /{ }^{32} \mathrm{~S}$ in standards are the result of variations in IMF rather than sample heterogeneity; nevertheless, this is a common method of fractionation correction in TIMS and MC-ICPMS which strive for much higher precision than SIMS (cf. Marechal et al., 1999), and so it is useful to compare its results to the Regression Method. The main difference between these two methods is that somewhat larger total uncertainties in $\delta^{33} \mathrm{~S}$ and $\delta^{34} \mathrm{~S}$ (but not $\Delta^{33} \mathrm{~S}$ ) can arise in the Regression Method if there are mass-dependent drifts in the IMF over the session that are not corrected for; if instrumental drift is minimal or absent, as in the data reported here, the two data reduction methods give identical results.

\subsection{Mass-dependent fractionation in SIMS and nature}

It should be remarked here that there are several different ways to calculate the exponent for multi-isotope fractionation ( $\beta_{I M F}$ for instrumental fractionation, $\beta_{M D F L}$ for natural fractionation, Young et al., 2002), and that these two $\beta$ values are not likely to be described by the same value. Indeed, the most commonly used expression for $\beta_{I M F}$ gives a value of 0.50831 for ${ }^{32} \mathrm{~S}_{-}{ }^{33} \mathrm{~S}-{ }^{34} \mathrm{~S}$ (and that is the value we have used here), whereas equilibrium isotopic fractionation in nature is expected to follow a law with $\beta_{M D F L}=0.51588$, and kinetic sulfur isotope fractionation is characterized by $\beta_{M D F L}=0.50453$ (Young et al., 2002). In theory, the difference between $\beta_{I M F}$ and $\beta_{M D F L}$ has potential to create artificial isotopic anomalies in $\Delta^{33} \mathrm{~S}$ if the measured sulfur isotopic compositions are far removed from the true ratios, i.e. when the magnitude of IMF is large; however in practice this is rarely the case in SIMS measurements of sulfur isotopes. In our study, this is largely because the efficiency of sulfur ionization is very high (better than $5 \%$ at low MRP) and the magnitude of the sulfur IMF in SIMS is correspondingly small, so the precise value of $\beta_{I M F}$ has no effect on the calculation or reproducibility of $\Delta^{33} \mathrm{~S}$ within our ability to measure it. This may not be the case, however, for isotope systems that display much larger magnitudes of instrumental mass fractionation; unfortunately, because SIMS often sputters an essentially infinite reservoir of analyte, there is usually a very small range in measured isotope ratios within individual analyses, and the regression of such data that could be used to determine $\beta_{I M F}$ is thus poorly constrained (note this is not the case for TIMS or MC-ICPMS, see Marechal et al., 1999).

\subsection{External reproducibility and standard homogeneity}

In summary, the results of the two 2-day sessions described here demonstrate that the NanoSIMS 50L is capable of high-precision stable isotope measurements at a level that is useful for geochemical and cosmochemical studies. Overall, our data show a long-term $2 \sigma$ reproducibility of $\pm 1.1 \%$ ofor $\delta^{34} S$ and $\pm 0.7 \%$ ofor $\delta^{33} S$ when averaged over all standards. However, there are patterns in the reproducibility of our data that indicate some of our standards are more homogeneous than others. In particular, in increasing order Anderson pyrrhotite, Canyon Diablo troilite and Norilsk pentlandite are more heterogeneous when compared with Balmat pyrite, and this order is mirrored by standard data reported from other studies that use subsets of the same standards (Greenwood et al., 2000; Mojzsis et al., 2003; Papineau et al., 2005; Whitehouse et al., 2005) and is also a feature of the laser data of Crowe and Vaughan (1996). When considering the data for only the most demonstrably homogeneous of our standards - Balmat pyrite the overall reproducibility $(2 \sigma)$ is $\pm 0.8 \%$ ofor $\delta^{34} S$ and $\pm 0.5 \%$ o for $\delta^{33} \mathrm{~S}$; for mass-independent isotope effects, our reproducibility is $\pm 0.41 \%$ or for $\Delta^{33} \mathrm{~S}$ when averaged over all standards and assuming that all our standards fall on the same mass-dependent fractionation lines. The reproducibility for $\Delta^{33} \mathrm{~S}$ is slightly worse than some previous studies using the IMS1270 instrument (Farquhar et al., 2002; Mojzsis et al., 2003; Papineau et al., 2005, 2007; Kamber and Whitehouse, 2007; Whitehouse et al., 2005; Phillippot et al., 2007) or the IMS 1280 ion probe (Kozdon et al., 2010; Williford et al., 2011; Reuschel et al., 2012; Wacey et al., 2010, 2011; Cabral et al., 2013; Whitehouse, 2013), however we make note here that we have used ${ }^{32} \mathrm{~S}$ ion beam intensities that are 2-4 times lower than these prior studies, and that higher beam intensities resulting in improved signal/baseline ratios for ${ }^{33} \mathrm{~S}$ can be expected to result in improvements in isotopic reproducibility. The volume of analyte removed by NanoSIMS is similar to, or somewhat smaller than, the volume sputtered by the IMS 1270 or 1280 ion microprobes, which is another factor to consider if the sample of interest is very limited such as in the case of extraterrestrial sample return. At the same time, for the NanoSIMS instrument there are a number of instrumental and sample-related factors that must be controlled in order to permit the instrument to achieve improvements in reproducibility over shorter time periods, and can thus achieve better performance if standardization and analytical sessions are subdivided accordingly.

\subsection{Factors affecting measurement reproducibility}

As in TIMS and MC-ICPMS applications in other isotope systems, attention to Faraday amplifier gains is an important aspect of accurate sulfur isotope analyses using the NanoSIMS 50L. Well-aligned delivery of the ion beams into the detectors is also important; the NanoSIMS operates in static multicollection mode with NMR-based magnet regulation, and the secondary ion beam is scanned horizontally across each detector's exit slit by means of dedicated pairs of electrostatic deflectors (Pd) located just in front of each exit slit (Fig. 1). Positioning of the moveable Faraday Cups to minimize this deflection at the peak center is desirable. In addition, the alignment of the ion beams with the exit slits in the vertical direction is also key, this is checked with a scan of C4X and it is important that the focusing elements LF4, LF5 and Q are tuned so that mass resolution is optimized, and a flat-topped peak is produced for all detectors on a scan of $C 4 \mathrm{x}$, and that a $\mathrm{C} 4 \mathrm{x}$ value is chosen that is located well onto the peak flat for all the isotopes of interest.

The NanoSIMS produces a very small diameter primary beam on the sample, and as a consequence the beam that is presented to the entrance slit is also very small. Stray magnetic fields surrounding the instrument produce subtle mass fractionation of the sputtered ion beam that enters the mass spectrometer. This is corrected by a pair of Helmholz coils (Bf-vert and Bf-horiz) located between the electron gun and the entrance slit, and attention to careful tuning of these coils is required so that all three sulfur isotopes are centered on the entrance slit. Finally, automated secondary ion beam centering (SIBC) at the entrance slit is required at the beginning of every analysis so that the sputtered ions are delivered to the mass spectrometer in a highly reproducible way.

The final instrumental factor influencing isotopic reproducibility is the $Z$ position of the sample stage. The close proximity of the sample surface to the EOW-EOP-EOS coaxial lens stack is key to achieving a small primary ion beam, but it also makes the instrument sensitive to small variations in the nominal $400 \mu \mathrm{m}$ distance between the sample surface and the coaxial lens. The lens EOS can be tuned to compensate for changes in sample height at different sample locations, but changes in EOS tuning result in downstream changes to the focusing of the sputtered ion beam in the mass spectrometer. To test the influence of these effects on isotopic reproducibility, we made repeat alternating measurements of two different grains of Balmat pyrite located on adjacent holes of an 8-hole sample holder (Fig. 3 and Table 1). For the first set of measurements, we kept the $Z$ coordinate of the sample stage fixed, and performed automated scanning of EOS voltage prior to each measurement, in which the chosen EOS value was the one that maximized the intensity of ${ }^{32} \mathrm{~S}$. This resulted in a bimodal population of measured ratios for the two grains analyzed, and a combined 
A

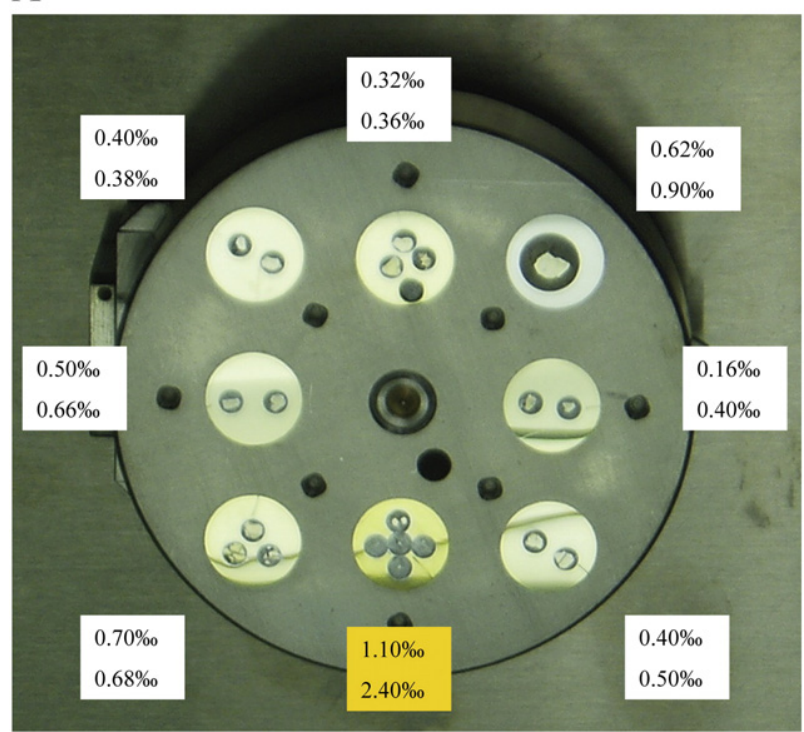

B

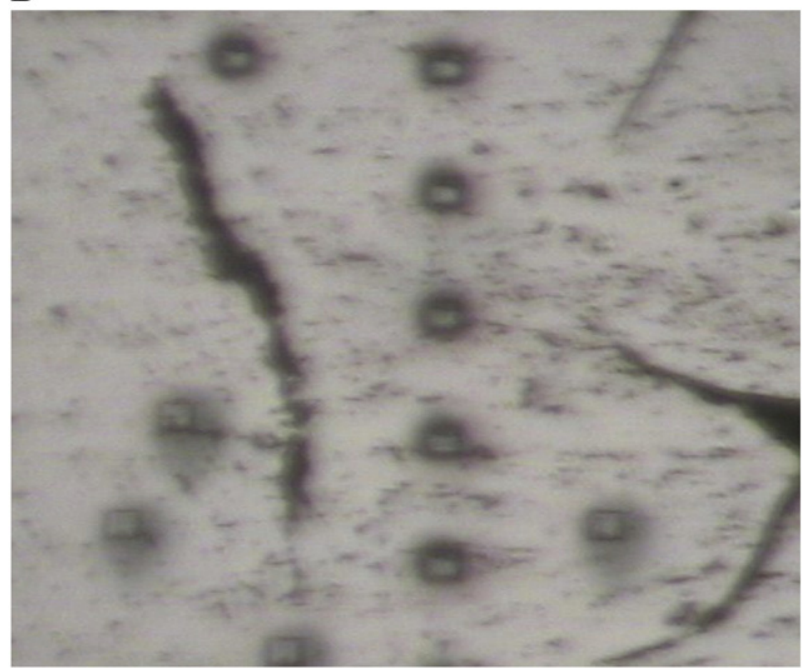

Fig. 3. (A) Photograph of a NanoSIMS 8-hole sample holder, with each hole containing a polished grain of Balmat pyrite. This sample was used to perform longer-term and sample-dependent tests of sulfur isotope reproducibility; labels show the external $2 \sigma$ reproducibility for $\delta^{34} \mathrm{~S}, \delta^{33} \mathrm{~S}$ and $\Delta^{33} \mathrm{~S}$ for each grain mount. Overall reproducibility for data obtained on these samples was $\pm 0.7 \%$ 。 $(2 \sigma)$ for $\delta^{34} S, \pm 0.5 \%$ 。 $(2 \sigma)$ for $\delta^{33} S$ and $\pm 0.32 \%$ or for $\Delta^{33} \mathrm{~S}$, demonstrating that careful control over instrumental tuning, sample Z positioning, and sample polish quality are required in order to obtain high-precision isotope data from the NanoSIMS $50 \mathrm{~L}$. (B) Ruttan pyrite grain from the central lowermost position of the holder in A (with stats highlighted in yellow); poor ploish (pitting) is correlated with sub-optimal data reproducibility.

reproducibility of $\pm 1.87 \%$ 。 $(2 \sigma)$. For the second set of measurements, we kept the EOS voltage fixed and found the unique $\mathrm{Z}$ coordinate for each pyrite grain that maximized the intensity of ${ }^{32} \mathrm{~S}$; the resulting reproducibility for the subsequent set of alternating measurements was $\pm 0.30 \%$ o $(2 \sigma)$, a factor of six improvement in isotopic reproducibility. This shows clearly that attention to determining the optimal $\mathrm{Z}$ coordinate of each analysis area contributes to large improvements in isotopic reproducibility.

As a further test of the ability to control this source of analytical uncertainty, we prepared eight different polished grains of Balmat pyrite, and mounted them in an 8-hole sample holder (Fig. 3). The optimal Z coordinate for each grain was determined by maximization of the ${ }^{32} \mathrm{~S}$ count rate on a test crater. A single analysis on each of the eight grains was subsequently performed, and this cycle was repeated five times (analyses "Tour8c-" in Table 1). The overall reproducibility for the entire population of data was $\pm 0.7 \%$ 。 $(2 \sigma)$ for $\delta^{34} S, \pm 0.5 \%$ 。 $(2 \sigma)$ for $\delta^{33} S$ and $\pm 0.32 \%$ for $\Delta^{33}$ S. There was no correlation of the average measured ratio or the reproducibility with the grain's location on the sample holder; the only exception was the data for the grain located in the South position of the sample holder, which had a noticeably worse quality of surface polish than all the other grains. The influence of sample polish quality and surface relief has been observed previously in SIMS studies of oxygen isotopes (Whitehouse and Nemchin, 2009; Kita et al., 2009, 2011). A repeat of this experiment excluding this grain, and making 10 repeat cycles around the other grains, showed a similar level of reproducibility (analyses "Tour12c-" in Table 1). This test demonstrates that optimization of each grain's Z coordinate is possible over the entire region of the NanoSIMS sample holder, shows that there are no biases related to specific positions of the sample holder on the sample stage, and that sample polish quality is also an important factor in determining the reproducibility of this method on the NanoSIMS.

Overall, the data for the sulfur isotope standards show a welldefined relationship among ${ }^{32} \mathrm{~S},{ }^{33} \mathrm{~S}$ and ${ }^{34} \mathrm{~S}$ (Figs. 4, 5) with the data defining a MDFL with slopes that are consistent with prior studies using both conventional gas-source mass spectrometry and SIMS, and this high level of external reproducibility is maintained between the two sessions separated by a re-tuning of the primary beam (Figs. 6 \& 7). These data demonstrate that with careful attention to instrument tuning, sample holder height and polish quality, the NanoSIMS is capable of high-precision sulfur isotope measurements required for the study of the terrestrial sulfur cycle.

\section{Sulfur isotope data from the Neoarchean Abitibi Supracrustal Terrain}

In addition to the standard data, we tested the analytical technique on a small selection of sulfides extracted from metasedimentary rocks collected from the Neoarchean Abitibi Supracrustal terrane in Ontario, Canada. These samples were selected because of their potential to preserve mass-independently fractionated sulfur isotopes, and were thus a useful test of the NanoSIMS analytical technique for $\Delta^{33} \mathrm{~S}$ analysis.

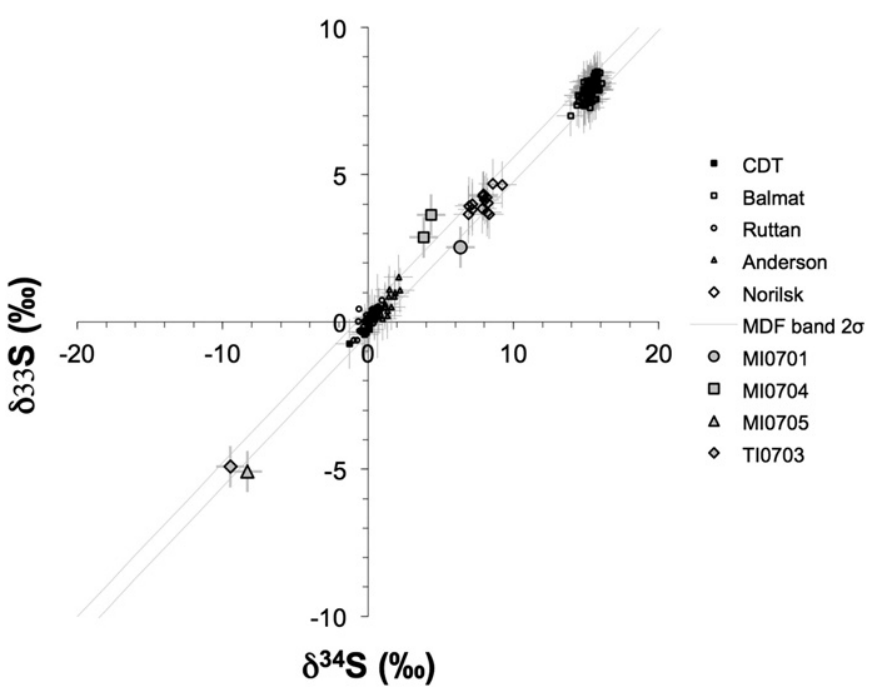

Fig. 4. Sulfur three-isotope plots for ${ }^{32} \mathrm{~S}^{3}{ }^{33} \mathrm{~S}-{ }^{34} \mathrm{~S}$ obtained in Session 1 . Standard grains are identified in the legend, while sulfides from banded iron formations (BIFs; MI0701, MI0704, MI0705, TI0703) are shown in large open symbols. Parallel thin lines show the 2 -sigma error bounds of the data around a terrestrial mass fractionation line (TFL) through the origin, with slopes of $0.5167( \pm 0.00432 \sigma)$ for ${ }^{32} \mathrm{~S}^{33}{ }^{33}-{ }^{34} \mathrm{~S}$. 


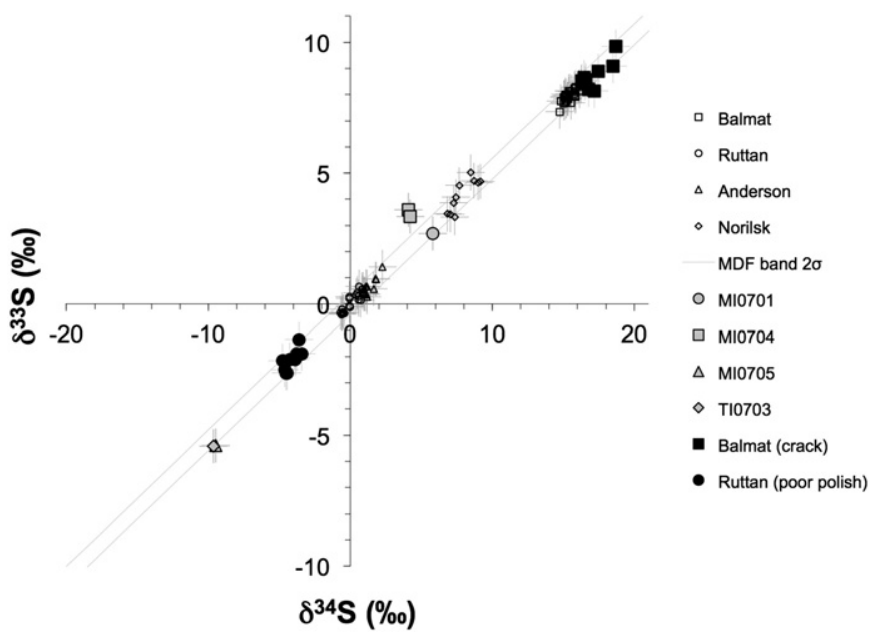

Fig. 5. Sulfur three-isotope plots for ${ }^{32} \mathrm{~S}-{ }^{33} \mathrm{~S}-{ }^{34} \mathrm{~S}$ obtained in Session 2 . The positive $\Delta^{33} \mathrm{~S}$ anomaly in BIF sulfide MI0704 is reproduced between the two sessions, as is the anomaly in MI0701 though it is within error of zero in Session 2. Data obtained for Balmat pyrite immediately adjacent to a crack in the grain, and for poorly polished Ruttan pyrite (Fig. 3B), are offset by $2-4 \%$ from their typical measured values.

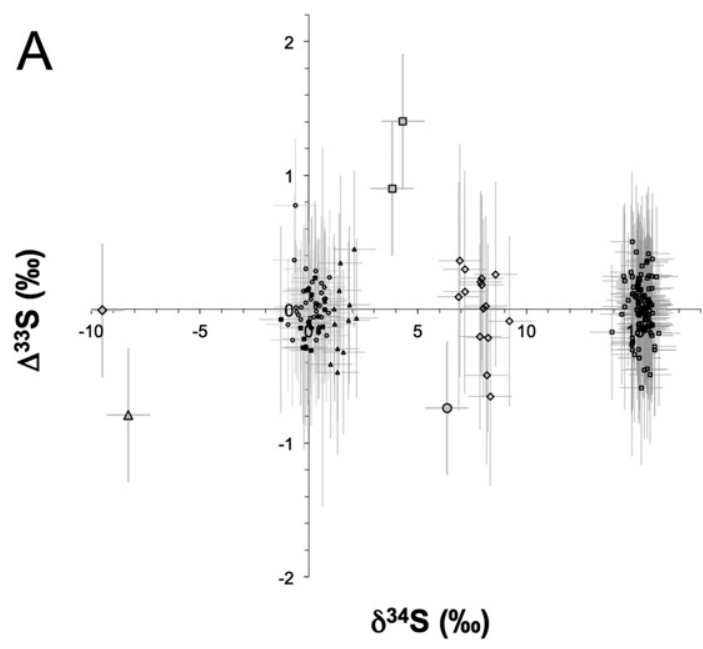

$\cdot \mathrm{CDT}$

- Balmat

- Ruttan

- Anderson

- Norilsk

omi0701

ㅁ.M10704

$\triangle \mathrm{M} 10705$

॰T10703

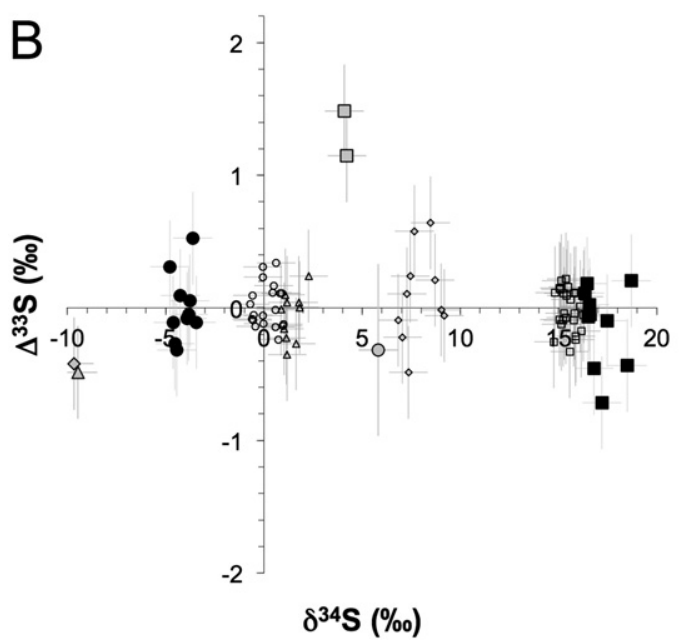

口Balmat

o Ruttan

$\triangle$ Anderson

- Norilsk

OMI0701

口M10704

$\triangle \mathrm{MI0705}$

○T10703

-Balmat (crack)

$\bullet$ Ruttan (poor polish)

\section{$\delta^{34} S(\%)$}

Fig. 6. $\Delta^{33} \mathrm{~S}$ plots for BIF samples and standards in Session 1 (A) and Session (B). In this diagram, the mass-dependent fractionation line (MDFL) is horizontal with $\Delta^{33} \mathrm{~S}=0$. In Session 2 (panel B), Balmat pyrite analyses adjacent to cracks, and Ruttan pyrite analyses on a poorly polished grain, display sub-optimal reproducibility of $\Delta^{33} \mathrm{~S}$.
Four different types of metasedimentary rocks were analyzed from the Neoarchean Michipicoten Greenstone Belt (Turek et al., 1982, 1984):

- Siderite facies iron formation (MI0701) usually massive with finely disseminated pyrite, deposited in shallow marine environment (Hofmann et al., 1991). Sample collected from the Sir James Dunn sid-

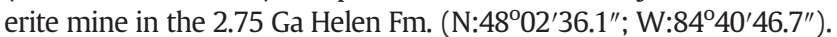

- Black shale with cm-size pyrite concretions (MI0704), immediately next yo the Sir James Dunn mine and part of the 2.75 Ga Helen Fm. (N:48 02'39.6"; $\left.\mathrm{W}: 84^{\circ} 40^{\prime} 35.6^{\prime \prime}\right)$.

- Siderite-quartz-magnetite banded iron formation (BIF) (MI0705) with fine laminations, occasional beds of jaspilite, and euhedral sulfides. Sample collected from the Lucy mine (N:48 $02^{\prime} 25.6^{\prime \prime}$; $\left.\mathrm{W}: 84^{\circ} 41^{\prime} 01.8^{\prime \prime}\right)$.

- Jasper-magnetite-chert BIF (TI0703) from the ca. 2.723 Ga Deloro Group (Corfu et al., 1989). Sample collected from road side outcrop with abundant quartz veins cross-cutting bedding of the BIF (N:48 02'25.6"; $\left.\mathrm{W}: 84^{\circ} 41^{\prime} 01.8^{\prime \prime}\right)$.

Our data for all samples during both sessions show a large range of $\delta^{34} \mathrm{~S}$ values between -9.6 and $+6.3 \%$ o (Figs. $4-6$; Table 2 ), which is larger than the range reported from the nearby Neoarchean Kidd Creek volcanogenic massive sulfide deposit and associated sulfidic turbidites with a range between -4.3 and $+5.5 \%$ o (Jamieson et al., 2012) and black shales with a range between -1.9 and $+5.0 \%$ (Kurzweil et al., 2013). We note however that there are sulfides in the Neoarchean Tumbiana Fm. of Australia with a range of $\delta^{34} \mathrm{~S}$ values between -5.7 and $+2.8 \%$ o (Thomazo et al., 2009) and in the Manjeri Fm. of Zimbabwe that have a range of $\delta^{34} \mathrm{~S}$ values between -15.2 and $+2.4 \%$, which have been attributed to microbial sulfate reduction (Thomazo et al., 2013).

Measured $\Delta^{33} S$ values range between -0.8 and $+1.5 \%$ (Fig. 6), which clearly resolves mass independently fractionated sulfur isotope compositions, and thus an atmospheric sulfur signature incorporated in the marine sulfur cycle. This range is similar to the range of $\Delta^{33} \mathrm{~S}$ values measured for Kidd Creek black shales to be between -1.0 and $+3.8 \%$ (Kurzweil et al., 2013) and to that of the Manjeri and Cheshire Fm. Sedimentary sulfides between -0.58 and $+1.17 \%$ o (Thomazo et al., 2013).

These data point to kinetic processes such as microbial sulfate reduction under non-limiting sulfate concentrations (Habicht et al., 2002). The $\Delta^{33} \mathrm{~S}$ values show significant enrichments in ${ }^{33} \mathrm{~S}$ and resolvable MIF. Our results suggest that at least some sulfur in the analyzed sulfides cycled through the anoxic atmosphere where it was subjected to photolysis in gas phase reactions prior to deposition in the marine system (Farquhar et al., 2000). It is possible that the acquired MIF signature was later diluted by MDF sources of sulfate before microbial sulfate reduction and/or with MDF sources of sulfide after the microbial production of hydrogen sulfide. The incorporation of atmospheric MIF sulfur in these metasedimentary rocks is further demonstrated by the clearly resolvable $\Delta^{33} \mathrm{~S}$ values for pyrite concretions from the Helen black shale (MI0704) (Fig. 8). The presence of MIF in pyrite from siderite iron formations in conformable contact with organic-rich shales with diagenetic pyrite concretions that lack MIF is inconsistent with the hypothesis that thermochemical sulfate reduction was responsible for widespread MIF in the Archean (Watanabe et al., 2009) and opposite to what would be expected from such hydrothermally-influenced organic-rich systems.

\section{Conclusions}

We have demonstrated that the NanoSIMS 50L ion microprobe is capable of high-precision measurements of multiple isotopes of sulfur, to a precision sufficient to resolve mass-independent variations in ${ }^{33} \mathrm{~S}$ $\left(\Delta^{33} \mathrm{~S}\right)$ on the order of $0.4 \%$ 。 $(2 \sigma)$, with a spatial resolution of $15 \mu \mathrm{m}$, similar to the precision and accuracy of large radius SIMS instruments. Data on pyrite grains from the Abitibi Supracrustal Terrain show large 
variations in $\delta^{34} \mathrm{~S}$ values between -9.6 and $+6.3 \%$, and corresponding $\Delta^{33} \mathrm{~S}$ values between -0.8 and $+1.5 \%$, in line with previous measurements of Neoarchean sulfides, including from the Abitibi terrane. Our results indicate that microbial sulfate reduction was widespread in the Neoarchean, and demonstrate that atmospheric sulfur affected by mass-independent fractionation of ${ }^{33} \mathrm{~S}$ due to photochemical reactions was incorporated in the Neoarchean marine sulfur cycle. These initial multiple sulfur isotope results, obtained on the NanoSIMS $50 \mathrm{~L}$ instrument for the first time, demonstrate the ability of this next generation of SIMS instrument to address important problems in stable isotope geochemistry and cosmochemistry that require precise stable isotope data at high spatial resolution.

\section{Acknowledgments}

The purchase of the NanoSIMS 50L was made possible by funding from the Carnegie Institution of Washington and NASA. We thank the engineers of Cameca Instruments SA for continued collaborative efforts to improve the ability of the NanoSIMS to produce high-precision isotope data. The development of the NanoSIMS 50L was supported by the Carnegie Institution of Washington and the NASA Astrobiology Institute (Grant No. NNA04CC09A). DP wishes to acknowledge University College London, the Carnegie Institution of Washington and NASA (Grant No. NNG04GH08G) for support.

\section{References}

Cabral, R.A., Jackson, M.G., Rose-Koga, E.R., Koga, K.T., Whitehouse, M.J., Antonelli, M.A., Farquhar, J., Day, J.M.D., Hauri, E.H., 2013. Anomalous sulphur isotopes in plume lavas reveal deep mantle storage of Archaean crust. Nature 496, 490-494.

Cates, N.L., Mojzsis, S.J., 2006. Chemical and isotopic evidence for widespread Eoarchean metasedimentary enclaves in southern West Greenland. Geochim. Cosmochim. Acta 70, 4229-4257.

Clayton, R.N., 2003. Oxygen isotopes in the solar system. Space Sci. Rev. 106 (1-4), 19-32.

Corfu, F., Kroch, T.E., Kwok, Y.Y., Jensen, L.S., 1989. U-Pb zircon geochronology in the southwestern Abitibi greenstone belt, Superior Province. Can. J. Earth Sci. 26 (9), 1747-1763.

Crowe, D.E., Vaughan, R.G., 1996. Characterization and use of isotopically homogeneous standards for in situ laser microprobe analysis of S-34/S-32 ratios. Am. Mineral. 81 (1-2), 187-193.

Ding, T., et al., 2001. Calibrated sulfur isotope abundance ratios of three IAEA sulfur isotope reference materials and V-CDT with a reassessment of the atomic weight of sulfur. Geochim. Cosmochim. Acta 65 (15), 2433-2437.

Evans, K.A., Tomkins, A.G., Cliff, J., Fiorentini, M.L., 2014. Insights into subduction zone sulfur recycling from isotopis analyis of eclogite-hosted sulfides. Chem. Geol. 365, 1-19.

Farquhar, J., Wing, B.A., 2003. Multiple sulfur isotopes and the evolution of the atmosphere. Earth Planet. Sci. Lett. 213, 1-13.

Farquhar, J., Bao, H.M., Thiemens, M., 2000. Atmospheric influence of Earth's earliest sulfur cycle. Science 289 (5480), 756-758.

Farquhar, J., et al., 2002. Mass-independent sulfur of inclusions in diamond and sulfur recycling on early earth. Science 298 (5602), 2369-2372.

Fischer, et al., 2014. PNAS.

Greenwood, J.P., Mojzsis, S.J., Coath, C.D., 2000. Sulfur isotopic compositions of individual sulfides in Martian meteorites ALH84001 and Nakhla: implications for crust-regolith exchange on Mars. Earth Planet. Sci. Lett. 184 (1), 23-35.

Habicht, K.S., Gade, M., Thamdrup, B., Berg, P., Canfield, D.E., 2002. Calibration of sulfate levels in the Archean ocean. Science 298 (5602), 2372-2374.

Hart, S.R., Zindler, A., 1989. Isotope fractionation laws: a test using calcium. Int. J. Mass Spectrom. Ion Process. 89, 287-301.

Hofmann, H.J., Sage, R.P., Berdusco, E.N., 1991. Archean stromatolites in Michipicoten group siderite ore at Wawa, Ontario. Econ. Geol. 86, 1023-1030.

Jamieson, J.W., Wing, B.A., Farquhar, J., Hannington, M.D., 2012. Neoarchean seawater sulphate concentrations from sulphur isotopes in massive sulphide ore. Nat. Geosci. 6, 61-64.

Kamber, B.S., Whitehouse, M.J., 2007. Micro-scale sulphur isotope evidence for sulphur cycling in the late Archean shallow ocean. Geobiology 5, 5-17.

King, H.E., Walters, C.C., Horn, W.C., Zimmer, M., Heines, M.M., Lamberti, W.A., Kliewer, C., Pottorf, R.J., Macloed, G., 2014. Sulfur isotope analysis of bitumen and pyrite associated with thermal sulfate reduction in reservoir carbonates at the Big Piney-La Barge production complex. Geochim. Cosmochim. Acta 134, 210-220.

Kita, N.T., Huberty, J.M., Kozdon, R., Beard, B.L., Valley, J.W., 2011. High-precision SIMS oxygen, sulfur and iron stable isotope analyses of geological materials: accuracy, surface topography and crystal orientation. Surf. Interface Anal. 43, 427-431.

Kita, N.T., Ushikubo, T., Fu, B., Valley, J.W., 2009. High precision SIMS oxygen isotope analysis and the effect of sample topography. Chem. Geol. 264, 43-57.

Kozdon, R., Kita, N.T., Huberty, J.M., Fournelle, J.H., Johnson, C.A., Valley, J.W., 2010. In situ sulfur isotope analysis of sulfide minerals by SIMS: precision and accuracy with application to thermometry of 3.5 Ga Pilbara cherts. Chem. Geol. 275, 243-253.
Kurzweil, F., Claire, M., Thomazo, C., Peters, M., Hannington, M., Strauss, H., 2013. Atmospheric sulfur rearrangement 2.7 billion years ago: evidence for oxygenic photosynthesis. Earth Planet. Sci. Lett. 366, 17-26.

Mahon, K.I., 1996. The new "York" regression: application of an improved statistical method to geochemistry. Int. Geol. Rev. 38, 293-303.

Marechal, C.N., Telouk, P., Albarede, F., 1999. Precise analysis of copper and zinc isotopic compositions by plasma-source mass spectrometry. Chem. Geol. 156 (1-4), 251-273.

McLoughlin, N., Grosch, E.G., Kilburn, M.R., Wacey, D., 2012. Sulfur isotope evidence for a Paleoarchean subseafloor biosphere, Barberton, South Africa. Geology 40, 1031-1034

Messenger, S., Stadermann, F.J., Floss, C., Nittler, L.R., Mukhopadhyay, S., 2003. Isotopic signatures of presolar materials in interplanetary dust. Space Sci. Rev. 106 (1-4), 155-172.

Mittlefehldt, D.W., Clayton, R.N., Drake, M.J., Righter, K., 2008. Oxygen isotopic composition and chemical correlations in meteorites and the terrestrial planets. Oxygen Solar Syst. 68, 399-428.

Mojzsis, S.J., Coath, C.D., Greenwood, J.P., McKeegan, K.D., Harrison, T.M., 2003. Massindependent isotope effects in Archean ( 2.5 to $3.8 \mathrm{Ga}$ ) sedimentary sulfides determined by ion microprobe analysis. Geochim. Cosmochim. Acta 67, 1635-1658.

Ono, S., Wing, B., Rumble, D., Farquhar, J., 2006. High precision analysis of all four stable isotopes of sulfur (S-32, S-33, S-34 and S-36) at nanomole levels using a laser fluorination isotope-ratio-monitoring gas chromatography-mass spectrometry. Chem. Geol. 225 (1-2), 30-39.

Ono, S., et al., 2003. New insights into Archean sulfur cycle from mass-independent sulfur isotope records from the Hamersley Basin, Australia. Earth Planet. Sci. Lett. 213 (1-2) 15-30.

Papineau, D., Mojzsis, S.J., 2006. Mass-independent fractionation of sulfur isotopes in sulfides from the pre-3770 Ma Isua Supracrustal Belt, West Greenland. Geobiology 4 (4), 227-238.

Papineau, D., Mojzsis, S.J., Coath, C.D., Karhu, J.A., McKeegan, K.D., 2005. Multiple sulfur isotopes of sulfides from sediments in the aftermath of Paleoproterozoic glaciations. Geochim. Cosmochim. Acta 69 (21), 5033-5060.

Papineau, D., Mojzsis, S.J., Schmitt, A.K., 2007. Multiple sulfur isotopes from Paleoproterozoic Huronian interglacial sediments and the rise of atmospheric oxygen. Earth Planet. Sci. Lett. 255 (1-2), 188-212.

Paterson, B.A., Riciputi, L.R., McSween, H.Y., 1997. A comparison of sulfur isotope ratio measurement using two ion microprobe techniques and application to analysis of troilite in ordinary chondrites. Geochim. Cosmochim. Acta 61 (3), 601-609.

Phillippot, P., van Zuilen, M., Lepot, K., Thomazo, C., Farquhar, J., van Kranendonk, M.J. 2007. Early Archaean microorganisms preferred elemental sulfur, not sulfate. Science 317, 1534-1537.

Reuschel, M., Melezhik, V.A., Whitehouse, M.J., Lepland, A., Fallick, A.E., Strauss, H., 2012. Isotopic evidence for a sizable seawater sulfate reservoir at 2.1 Ga. Precambrian Res. 192-195, 78-88.

Riciputi, L.R., Paterson, B.A., Ripperdan, R.L., 1998. Measurement of light stable isotope ratios by SIMS: matrix effects for oxygen, carbon, and sulfur isotopes in minerals. Int. J. Mass Spectrom. 178 (1-2), 81-112.

Russell, W.A., Papanastassiou, D.A., Tombrello, T.A., 1978. Ca isotope fractionation on the Earth and other solar system materials. Geochim. Cosmochim. Acta 42, 1075-1090.

Thiemens, M.H., 1999. Atmosphere science - Mass-independent isotope effects in planetary atmospheres and the early solar system. Science 283 (5400), 341-345.

Thiemens, M.H., Heidenreich, J.E., 1983. The mass-independent fractionation of oxygen a novel isotope effect and its possible cosmochemical implications. Science 219 (4588), 1073-1075.

Thode, H.G., Monster, J., Dunford, H.B., 1961. Sulphur isotope geochemistry. Geochim. Cosmochim. Acta 25 (3), 159-174.

Thomazo, C., Ader, M., Farquhar, J., Philippot, P., 2009. Methanotrophs regulated atmospheric sulfur isotope anomalies during the Mesoarchean (Tumbiana Formation, Western Australia). Earth Planet. Sci. Lett. 279, 65-75.

Thomazo, C., Nisbet, E.G., Grassineau, N.V., Peters, M., Strauss, H., 2013. Multiple sulfur and carbon isotope compositions of sediments from the Belingwe Greenstone Belt (Zimbabwe): a biogenic methane regulation on mass independent fractionation of sulfur during the Neoarchean? Geochim. Cosmochim. Acta 121, 120-138.

Turek, A., Smith, P.E., van Schmus, W.R., 1982. Rb-Sr and U-Pb ages of volcanism and granite emplacement in the Michipicoten belt - Wawa, Ontario. Can. J. Earth Sci. $19,1608-1626$.

Turek, A., Smith, P.E., van Schmus, W.R., 1984. U-Pb zircon ages and the evolution of the Michipicoten plutonic-volcanic terrane of the Superior Province, Ontario. Can. J. Earth Sci. 21, 457-464.

Valley, J.W., Cole, D.R., 2001. Stable isotope geochemistry. Rev. Mineral. 43 (661 pp.).

Valley, J.W., Taylor, H.P., O'Neil, J.R., 1986. Stable isotopes in high-temperature geologic processes. Rev. Mineral. 16.

Wacey, D., Kilburn, M.R., Saunders, M., Cliff, J., Brasier, M.D., 2011. Microfossils of sulphurmetabolizing cells in 3.4-billion-year-old rocks of Western Australia. Nat. Geosci 4 (10), 698-702.

Wacey, D., McLoughlin, N., Whitehouse, M.J., Kilburn, M.R., 2010. Two coexisting sulfur metabolisms in ca. $3400 \mathrm{Ma}$ sandstone. Geology 38, 1115-1118.

Watanabe, Y., Farquhar, J., Ohmoto, H., 2009. Anomalous fractionations during thermochemical sulfate reduction. Science 324, 370-373.

Whitehouse, M.J., 2013. Multiple sulfur isotope determination by SIMS: evaluation of reference sulfides for $\Delta^{33} \mathrm{~S}$ with observations and a case study on the determination of $\Delta^{36}$ S. Geostand. Newslett. 37, 19-33.

Whitehouse, M.J., Nemchin, A.A., 2009. High precision, high accuracy measurement of oxygen isotopes in a large lunar zircon by SIMS. Chem. Geol. 261, 32-42.

Whitehouse, M.J., Kamber, B.S., Fedo, C.M., Lepland, A., 2005. Integrated Pb- and S-isotope investigation of sulphide minerals from the early Archaean of Southwest Greenland. Chem. Geol. 222, 112-131. 
Williford, K.H., van Kranendonk, M.J., Ushikubo, T., Kozdon, R., Valley, J., 2011. Constraining atmospheric oxygen and seawater sulfate concentrations during Paleoproterozoic glaciation: in situ three-isotope microanalysis of pyrite from the Turee Creek Group, Western Australia. Geochim. Cosmochim. Acta 75, 5686-5705.

Xiao, S., Schiffbauer, J.D., McFadden, K.A., Hunter, J., 2010. Petrographic and SIMS pyrite sulfur isotope analyses of Ediacaran chert nodules: implications for microbial processes in pyrite rim formation, silicification, and exceptional fossil preservation. Earth Planet. Sci. Lett. 297, 481-495.

Young, E.D., Galy, A., Nagahara, H., 2002. Kinetic and equilibrium mass-dependent isotope fractionation laws in nature and their geochemical and cosmochemical significance. Geochim. Cosmochim. Acta 66 (6), 1095-1104. 